\title{
e-Phaïstos
}

e-Phaïstos

Revue d'histoire des techniques / Journal of the history

of technology

IX-2 | 2021

Quel objet pour quel musée?

\section{L'Académie François Bourdon et la valorisation des patrimoines industriels}

Bâtiments, objets, archives de l'industrie

The François Bourdon Academy and the Valorization of Industrial Heritage:

Buildings, Objects and Archives of industry

\section{Ivan Kharaba}

\section{OpenEdition \\ Journals}

Édition électronique

URL : https://journals.openedition.org/ephaistos/9400

DOI : 10.4000/ephaistos.9400

ISSN : 2552-0741

Éditeur

IHMC - Institut d'histoire moderne et contemporaine (UMR 8066)

\section{Référence électronique}

Ivan Kharaba, «L'Académie François Bourdon et la valorisation des patrimoines industriels », e-

Phaïstos [En ligne], IX-2 | 2021, mis en ligne le 26 octobre 2021, consulté le 28 octobre 2021. URL

http://journals.openedition.org/ephaistos/9400 ; DOI : https://doi.org/10.4000/ephaistos.9400

Ce document a été généré automatiquement le 28 octobre 2021.

Tous droits réservés 


\title{
L'Académie François Bourdon et la valorisation des patrimoines industriels
}

\author{
Bâtiments, objets, archives de l'industrie \\ The François Bourdon Academy and the Valorization of Industrial Heritage: \\ Buildings, Objects and Archives of industry
}

\section{Ivan Kharaba}

1 L'Académie François Bourdon, association loi 1901, a été créée en 1985 par d'anciens cadres de l'usine du Creusot. En effet, au moment de la liquidation de Creusot-Loire, d'anciens salariés se préoccupent de la sauvegarde des archives de la société qui renferment non seulement les archives produites entre 1970 et 1985, mais aussi les fonds plus anciens remontant à la création de la société Schneider en 1837. Quelques documents portent même sur la période précédente, depuis le début de la Fonderie Royale et de l'histoire de la métallurgie au Creusot, en 1782.

2 Le rôle des bénévoles pour la sauvegarde de ces archives a été important. Ce sont eux qui ont obtenu le dépôt des documents à l'Académie François Bourdon, puis leur classement en archives historiques par le ministère de la Culture. Ce fonds initial comprend environ 3000 mètres linéaires de dossiers et registres, 80000 photographies et 100000 plans. Il faut y adjoindre une bibliothèque historique spécialisée en science et technique d'environ 25000 volumes ainsi qu'une collection d'objets et maquettes représentant environ 750 pièces.

3 Forte de son fonds initial, l'Académie François Bourdon est vite reconnue comme centre d'archives industrielles. En 1999, les Archives Nationales du Monde du Travail versent les archives historiques du siège social des établissements Schneider (série 187 AQ), qui viennent ainsi compléter les fonds existants. En 2001, l'Union des Industries et Métiers de la Métallurgie confie à l'Académie la gestion de ses archives historiques à l'instar d'autres syndicats de salariés. Aujourd'hui, l'association gère aussi les archives des sociétés Fenwick, Framatome, Schneider Electric, Thermodyn... S'ajoutent enfin des 
dizaines d'autres versements d'entreprises et d'institutions comme ceux de l'International Iron \& Steel Institute ${ }^{1}$...

4 L'ensemble des archives historiques représente désormais environ 6500 mètres linéaires de dossiers, 520000 photographies, 150000 plans et une bibliothèque de 40000 volumes. À cela s'ajoute une collection d'objets historiques d'environ 1200 pièces dont une partie est présentée au centre d'interprétation du Pavillon de l'Industrie, sur le site du Château de la Verrerie, au Creusot.

Rares sont les villes qui s'identifient avec autant de force à une activité économique, comme Le Creusot à la sidérurgie et à la mécanique. Depuis 1782, Le Creusot vit de ces activités. Fait plus remarquable, pendant près de 150 années, elles étaient portées par une seule et même dynastie de maîtres de forges : les Schneider. Cette constante a doté la ville d'un patrimoine varié, de l'architecture aux archives industrielles, constitué sur une longue période, de la création de la fonderie royale en 1782 au début du XXI ${ }^{\mathrm{e}}$ siècle. Nous avons employé le passé, alors que nous devrions mettre en avant le présent, car Le Creusot reste une ville industrielle. C'est même l'une des rares villes d'importance en France à conserver autant d'emplois industriels directs. Dans son bassin d'emplois, environ $40 \%$ de la population active travaille dans l'industrie. Soulignons aussi l'importance significative du nombre de sites de production appartenant à des grands groupes internationaux comme Framatome, ArcelorMittal, Alstom, Safran, Haulotte, ou encore General Electric, avec son département Baker Hughes. Dans ce contexte, évoquer le patrimoine du Creusot consiste à faire référence à un patrimoine vivant et en constante évolution. La plupart des bâtiments industriels accueillent toujours des activités industrielles et, quand ce n'est plus le cas, ils ont été réinvestis, à l'instar du site universitaire Condorcet et de sa bibliothèque.

6 Le patrimoine industriel ne se limite pas à la seule architecture (Garçon 2000). Au Creusot, cette affirmation prend tout son sens. Le patrimoine se fait pluriel, à travers le bâti ou encore les collections d'objets et les archives. Cet article propose de faire découvrir les patrimoines que conserve l'Académie François Bourdon. Dans une première partie, nous déroulerons à grand trait le fil de l'histoire de la ville-usine. Nous développerons notre propos sur le patrimoine industriel à partir de deux axes, le premier consacré aux collections d'objets issus de l'industrie et leurs valorisations au sein du Pavillon de l'Industrie, le second relatif aux archives issues de l'histoire industrielle du Creusot.

\section{L'histoire industrielle du Creusot}

7 En 1782, dans un contexte où l'État est soucieux de rattraper le retard industriel de la France par rapport à l'Angleterre, François Ignace de Wendel, alors directeur des forges d'Indret, à proximité de Nantes, propose de s'approvisionner pour la fabrication de canons, non plus avec de la fonte anglaise, mais avec de la fonte au coke fabriquée en France, selon le procédé anglais. Après avoir visité plusieurs sites, il décide d'implanter sa fonderie au Creusot. Dans ce lieu qui n'est encore qu'un espace rural, le sous-sol renferme du charbon cokéfiable, les prix des terrains sont très peu élevés et il existe des mines de fer à proximité, dans la région de Couches (Passaqui 2006). Par ailleurs, Le Creusot est proche du futur canal du Centre dont le projet vient d'être validé. Ce canal relie la Loire, au niveau de Digoin, à la Saône, vers Chalon-sur-Saône. À une époque où les transports s'effectuent principalement par voie d'eau, il permet d'envisager 
l'expédition facile des productions sur les rives méditerranéennes et atlantiques et, inversement, d'assurer de plus amples approvisionnements.

Fig.1. Coulée de fonte à Indret (1864)

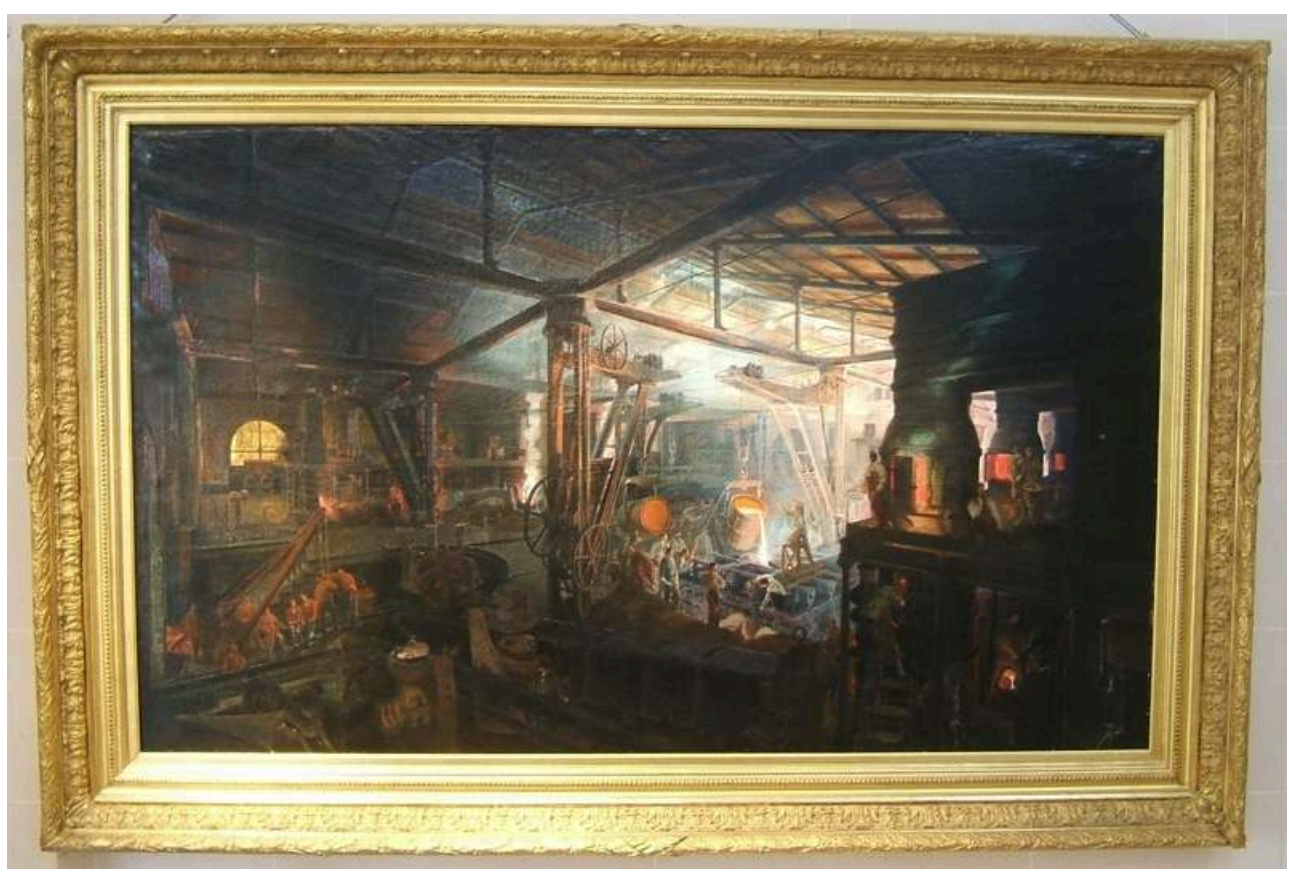

Dans ce tableau célèbre, François Bonhommé, le grand peintre de l'industrie du XIXe siècle, magnifie le corps-à-corps des équipes ouvrières avec la matière en fusion, sans négliger aucun détail technique.

Centre d'archives de l'Académie François Bourdon, La Verrerie, Le Creusot (Saône-et-Loire)

8 La fonderie est implantée dans la plaine des Riaux selon les plans de l'architecte de la marine royale Pierre Toufaire. Construite sur le modèle des châteaux de l'époque classique, elle accueille tout à la fois des halles de production et des logements ouvriers. Cette nouvelle fonderie représente un saut technologique remarquable. Au-delà de l'usage de la fonte au coke produite dans des hauts-fourneaux bien plus imposants que ceux qui marchent au charbon de bois, on utilise la nouvelle technologie de la machine à vapeur du type de celle de Watt pour faire fonctionner les soufflantes. Enfin, l'usine est dotée de voies ferrées, sur lesquelles bœufs et chevaux tirent des convois.

Dotée d'un équipement à la pointe de la technologie, la fonderie devient emblématique de la métallurgie française, comme l'illustrent plusieurs récits. L'usine connaît pourtant de nombreux déboires associés à une histoire chaotique. La fonte, de médiocre qualité, ne permet pas la réalisation de canons, ni sa transformation en fer. L'usine doit se contenter de fabriquer, avec sa propre fonte, des tuyaux, boulets, lests ou autres objets en fonte moulée.

10 Peu, voire non rentable, l'entreprise connaît très vite ses premières difficultés. Mais l'usine émerveille les contemporains et parait «toujours annoncer une sidérurgie entièrement nouvelle » (Bergeron $2001: 32$ ). Cette fascination, à laquelle il faut ajouter les outils de production et le savoir-faire local, explique que, malgré plusieurs faillites, l'établissement trouve à chaque fois de nouveaux repreneurs. Ainsi, après le départ de François-Ignace de Wendel, elle change de mains puis, en 1794, elle est réquisitionnée par le Comité de Salut Public. Par la suite, l'entreprise passe sous la coupe de plusieurs actionnaires majoritaires, comme l'entrepreneur bourguignon Jean-Baptiste Bureau, 
puis Jean François Chagot. En 1823, ce dernier s'associe à deux ingénieurs anglais, Manby et Wilson, alors propriétaires des forges de Charenton. Ils modernisent les usines du Creusot, en y installant des laminoirs et une forge à l'anglaise équipée de fours à puddler pour la décarburation de la fonte.

En 1828, Manby-Wilson rachètent aux Chagot la totalité des parts de la fonderie du Creusot qui est alors intégrée dans la Société anonyme des Usines, Forges, Fonderie du Creusot et de Charenton. Le surdimensionnement et le manque de fonds propres provoquent, en 1833, la faillite de la société. Après plusieurs péripéties, les établissements du Creusot sont mis aux enchères. Très vite, Adolphe Schneider s'y intéresse. Soutenu par le banquier Alexandre Seillière et son beau-père, Louis Boigues, industriel et homme d'affaires à l'origine des usines de Fourchambault, dans la Nièvre, il s'en porte acquéreur, en association avec Eugène, son jeune frère. C'est ainsi qu'est créée, le $1^{\text {er }}$ janvier 1837, la société Schneider Frères et Cie. Ses usines sont au Creusot pendant que son siège social est situé à Paris. La petite ville bourguignonne compte alors près de 2600 habitants dont 1200 ouvriers.

Dès leur arrivée, Adolphe Schneider et son frère Eugène remettent les usines en activité. Tout en gardant les productions traditionnelles en fonte et en fer comme, par exemple les rails, ils se lancent dans les constructions mécaniques avec la fabrication des machines à vapeur fixes et mobiles, comme les locomotives et bateaux. Dans ce cas, les coques sont fabriquées dans les chantiers navals qu'ils installent à Chalon-sur-Saône à partir de 1839. Les machines à vapeur construites au Creusot rencontrent très vite un immense succès. Elles participent au développement de l'Usine, à sa renommée internationale et à celle de la Société Schneider.

13 Au début des années 1840, la réussite de l'entreprise est assurée. Devenu le seul gérant après le décès accidentel de son frère en 1845 , Eugène Schneider fait croître l'usine du Creusot. Elle devient un des plus grands établissements industriels de France ainsi que le symbole de la puissance économique du pays. De ses ateliers sortent, outre des machines à vapeur, des tonnes de fer et tôles, des rails, des poutrelles métalliques pour la construction des ponts ou des charpentes... L'extension fulgurante des fabrications s'accompagne d'une augmentation significative du personnel. Le nombre d'ouvriers dépasse 9300 à la fin des années 1860. Parallèlement, l'usine s'agrandit. À partir de 1860, elle s'étend au-delà du site historique de la Plaine de Riaux pour commencer son extension vers le sud-est, avec la construction de la grande halle de la grande forge qui correspond désormais au site de la tôlerie d'Industeel.

14 Le développement de l'usine suscite aussi la croissance de la ville placée sous l'égide de l'entreprise et de sa politique sociale. Celle-ci s'accompagne de la construction des écoles, de maisons de retraite, en passant par la création d'une caisse de secours, la prise en charge des soins ou encore la construction de logements dans deux petites cités ouvrières. L'entreprise encourage et accompagne l'accession à la propriété. Ce "paternalisme schneidérien" a bien évidemment comme ambition de retenir une population ouvrière qualifiée auprès de l'usine. Au Creusot, île industrielle située entre les champs du Charolais et les contreforts du Morvan, la politique sociale patronale prend une dimension particulière, en raison de l'imbrication entre la ville et l'Usine. Le contrôle patronal s'exerce en dehors des ateliers, où l'ouvrier est placé sous la surveillance des employés, ceux-là mêmes qui les encadrent dans l'usine. Ce paternalisme, qui exclut toute organisation syndicale, prend fin au lendemain de la 
Seconde Guerre mondiale, époque où la gestion sociale entre dans l'ère du paritarisme caractéristique des Trente glorieuses.

Après le décès d'Eugène Schneider en 1875 , Henri, son fils, lui succède à la tête de la société familiale. Ainsi prend corps la dynastie de Maîtres de forges. Durant quatre générations, les gérants se succèdent de père en fils avec Eugène II, entre 1898 et 1942, puis Charles jusqu'en 1960. Charles ayant eu deux filles, la famille perd progressivement le contrôle de l'entreprise qui passe en 1970 dans le giron du groupe Empain ${ }^{2}$.

Au cours de la décennie 1860, Eugène Schneider implante au Creusot un laboratoire de recherche en métallographie dont l'activité est développée par son fils, afin de permettre la mise au point des aciers alliés. Parallèlement, il ouvre les productions vers la construction des premiers matériels électriques. Dès lors, Henri Schneider délaisse les produits à faible exigence technique pour se consacrer aux produits à haute valeur ajoutée. C'est l'époque du développement des blindages et de l'armement qui participent, avec les travaux publics et les constructions métalliques, à la réputation mondiale de la société Schneider. Sous sa gérance, l'usine poursuit son extension dans le prolongement de la grande forge, avec la construction, en 1888, de l'atelier d'artillerie Nord et, en 1897, de l'atelier d'artillerie Sud. Dans la ville, deux nouveaux quartiers voient le jour, l'un au sud, autour de l'église Saint-Henri, l'autre à l'est, près de l'Hôtel-Dieu (hôpital).

Eugène II Schneider, maître de forges de la troisième génération, poursuit l'œuvre engagée par ses ânés. Sous sa gérance, la société Schneider s'internationalise. Elle s'implante au Luxembourg, en Tchécoslovaquie ou encore en Pologne. En France, le développement passe par la construction ou le rachat de nombreux sites industriels : Bordeaux, Le Havre, Sète, Champagne-sur-Seine, La Londe-les-Maures, dans la continuité des acquisitions de mines comme La Machine trois décennies plus tôt... Véritable holding, la firme emploie 125000 salariés dans les années 1920. Les usines du Creusot en regroupent désormais plus de 20000 . La période est marquée par le développement de l'électricité. À côté des fabrications traditionnelles, de nouveaux produits voient le jour, comme les turbines à vapeur ou encore les compresseurs alternatifs puis centrifuges. Eugène Schneider modernise les usines du Creusot et poursuit leur extension. 
Fig.2. Prototype d'une génératrice à courant continu

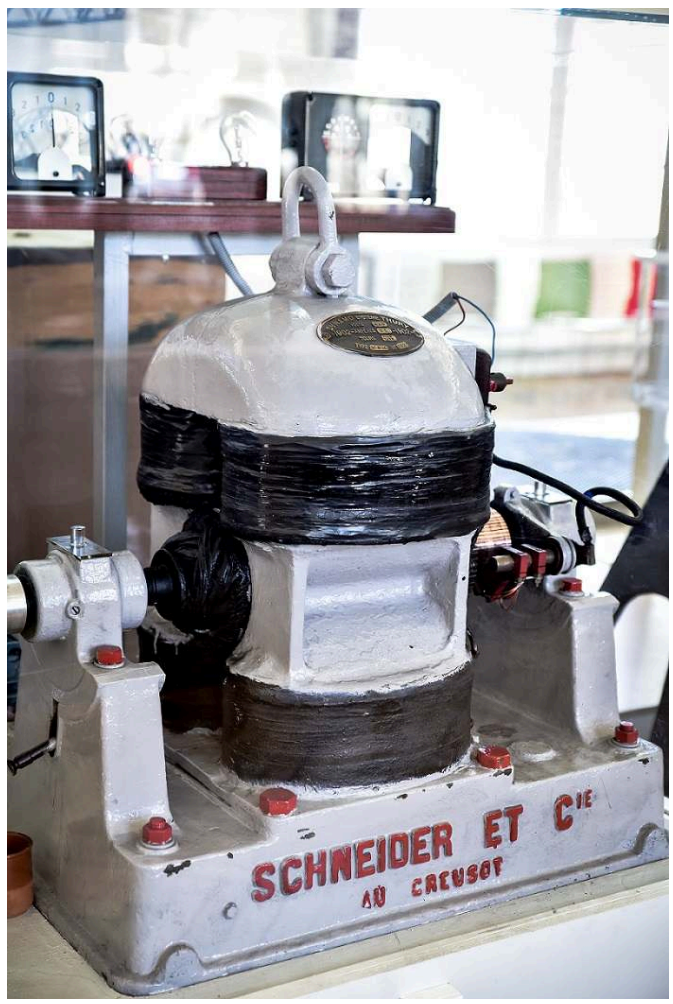

Il s'agit d'un prototype de dynamo système Thury datant de 1902.

Pavillon-Industrie HD-7255, (Franck-Juillot

18 Faute de place, les agrandissements se font sur le territoire des communes voisines. Au Breuil, sont implantés des ateliers de constructions mécaniques (1912), une nouvelle aciérie (1916) et des laminoirs (1918). Un peu plus loin, à Montchanin, une nouvelle fonderie et une cokerie sont inaugurées en 1919. Ces ateliers sont accompagnés par l'édification de nouvelles cités ouvrières, comme celle de la Mouillelongue, au Creusot, et celle des Quarts, à Montchanin.

Le début $\mathrm{du} \mathrm{XX} \mathrm{XX}^{\mathrm{e}}$ siècle constitue une période faste. L'usine du Creusot, plus que jamais symbole de la puissance industrielle de la France, devient aussi un témoignage de son excellence dans les matériels militaires. Des dirigeants du monde entier, présidents de la République, rois, princes, premiers ministres, délégations gouvernementales et bien d'autres encore, viennent régulièrement «porter leurs hommages au Creusot. » Pour accueillir ces délégations, Eugène Schneider entreprend la rénovation de l'ancienne manufacture des cristaux, qu'il transforme en château : le Château de la Verrerie.

Durant la Seconde Guerre mondiale, Le Creusot se trouve dans la zone occupée. Dès leur victoire, les troupes d'occupation allemande s'emparent de l'usine. À deux reprises, en 1942, puis en 1943, les alliés bombardent la ville et ses usines. La population civile est fortement touchée. Les funérailles des victimes du premier bombardement sont l'occasion pour les Creusotins d'entrevoir une dernière fois Eugène Schneider qui décède quelques semaines plus tard.

Charles Schneider poursuit l'œuvre engagée. Après les désastres de la Seconde Guerre mondiale, il entreprend la reconstruction des usines et les filialise sous le nom de Société des Forges et Ateliers du Creusot (SFAC). Portées par les marchés de 
reconstruction et de modernisation de la France, les usines tournent à plein régime. Percevant avant l'heure le développement possible de l'énergie nucléaire civile, Charles engage son groupe dans cette direction. Pour cela, en 1958, il crée, en association avec le groupe américain Westinghouse, une nouvelle société qu'il dénomme Francoaméricaine Atomique, autrement dit FRAMATOME. Cette dernière apporte au Creusot, dans les années 1970, l'élaboration de la grande chaudronnerie nucléaire.

La mort soudaine de Charles est ressentie comme un choc par la population creusotine. Mais la disparition du dernier des Schneider ne diminue en rien l'activité des usines locales. Pourtant, depuis les années 1950, un drame est en train de se préparer dans les industries métallurgiques et mécaniques françaises. Confrontées notamment au développement de la concurrence mondiale, elles sont contraintes à des restructurations importantes et indispensables. Dès le début des années 1960, des études sont lancées pour le regroupement de la Compagnie des Aciéries et Forges de la Loire (CAFL) avec la SFAC. N'y étant pas favorable, la société Schneider résiste jusqu'à ce que le gouvernement français l'impose en 1970, avec la création du groupe CreusotLoire. Dans cette nouvelle configuration, le chiffre d'affaires des usines du Creusot est en forte croissance. La réduction des effectifs - ils sont tombés à 5848 personnes en 1983 - conforte les résultats positifs. C'est la période du développement intensif des aciers spéciaux, de l'affinage en poche chauffante, de la coulée sous vide, de la chaudronnerie nucléaire, de l'augmentation de la fabrication des turbines à vapeur, de compresseurs... En parallèle, l'usine du Creusot cesse la fabrication des locomotives pour se recentrer sur les boggies, notamment ceux des $\mathrm{TGV}^{3}$.

Mais Le Creusot n'est pas Creusot-Loire. Depuis sa création, le groupe est confronté à d'importants problèmes financiers que viennent accentuer des acquisitions coûteuses et non rentables. À cela s'ajoute la situation économique nationale. Depuis la crise du milieu des années 1970, la France est entrée dans une phase de désindustrialisation majeure qui touche tous les secteurs industriels, y compris la métallurgie et les constructions mécaniques. À la situation fragile du groupe s'ajoutent les effets de la crise économique, ce qui conduit Creusot-Loire à la faillite et à sa liquidation en décembre 1984. Pour les Creusotins, c'est un véritable traumatisme et ceci d'autant plus qu'ils n'avaient pas vu ou n'avaient pas voulu voir le drame. Il allait en découler d'importantes et douloureuses restructurations.

Au moment de la liquidation, trois entreprises reprennent les actifs de Creusot-Loire au Creusot. Usinor Sacilor récupère la métallurgie, Jeumont Schneider prend le contrôle de la partie traction et Framatome, celui de la branche énergie. L'ensemble de ces activités se poursuit. Après d'importantes restructurations et plusieurs changements de propriétaires, l'industrie creusotine maintient son dynamisme en dépit de la crise économique : la métallurgie et l'activité tôlerie sont développées par Industeel, filiale du groupe ArcelorMittal ; l'activité liée à la fabrication des boggies par Alstom (branche transport); une partie de l'activité énergie a été reprise par NFMtechnologies qui fabriquait essentiellement des tunneliers, et l'autre, par Thermodyn (filiale du groupe américain General Electric) qui produit des compresseurs centrifuges et des petites turbines à vapeur ; Framatome enfin, qui a conservé la forge et une partie de la grosse mécanique, y usine la grosse chaudronnerie pour le nucléaire et les nouveaux marchés de la chimie.

Malgré la grande crise de la liquidation de Creusot-Loire, ou peut-être grâce à elle, l'industrie creusotine a su se restructurer et continuer ses activités. Certes, elle a 
changé d'échelle, mais avec un peu plus de 4000 emplois industriels, Le Creusot reste une des villes les plus industrieuses de France par rapport à sa population (23000 habitants). Autre nouveauté, l'industrie creusotine ne repose plus sur une monoactivité. Elle se répartit entre plusieurs grands groupes. Malgré ces changements importants, les principales productions actuelles sont dans la continuité de celles d'hier. Elles ont connu d'importantes évolutions technologiques, à la fois dans les produits et dans les méthodes. La permanence de cette industrie permet d'affirmer que le Creusot est un contre-exemple de la désindustrialisation que connaît la France.

\section{Les collections d'objets issues de l'industrie}

Les collections de l'Académie François Bourdon comptent de nombreux objets issus de l'industrie creusotine et de son histoire. Pour une partie d'entre elles, ces collections ont été constituées à la fin du XIX siècle, lors de la création du "Musée Schneider " (Kharaba 2009). À l'époque du paternalisme schneidérien triomphant, un nouveau vecteur s'offre au maître de forges pour moraliser et faire valoir la reconnaissance qu'on lui doit. Après l'école et l'ensemble des structures propres au paternalisme, le musée devient un outil de propagande à la gloire du Creusot, de son industrie et de ses maîtres d'alors.

Eugène Schneider fait constituer une collection pour doter son nouveau musée qu'il installe dans une des ailes du Château de la Verrerie. On y montre l'activité de la cristallerie à travers des objets. Une grande partie des collections porte sur des échantillons géologiques, l'industrie sidérurgique et les constructions mécaniques. Sont rassemblés des maquettes des fabrications Schneider ou celles illustrant des procédés de fabrication, des objets anciens que l'on récupère comme des vestiges: des tuyaux en fonte de conduites d'alimentation en eau de la ville de Paris fabriqués durant la Révolution Française; le "cylindre Wilkinson ", seule pièce rescapée de la machine à vapeur de type Watt installée au Creusot en 1782, lors de la création de la fonderie, ainsi que différentes maquettes de marteaux pilons, de locomotives, de machines marines. 
Fig.3. Maquette au $1 / 10^{\mathrm{e}}$ d'une machine marine à vapeur horizontale

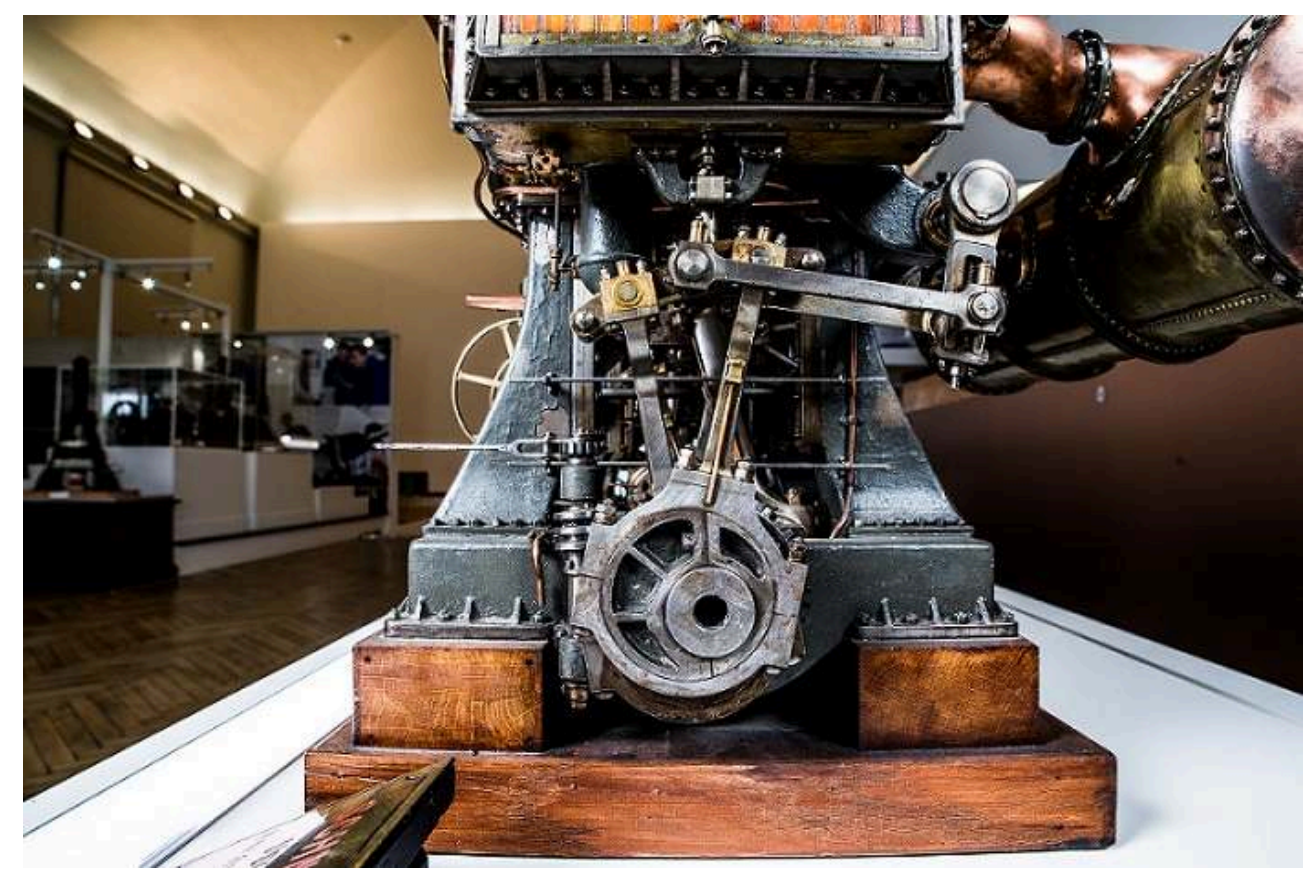

Machine à vapeur horizontale du croiseur-torpilleur "Wattignies", mis en service en 1891 et rayé des services en 1908 ( Classe Wattignies », Wikipedia).

Pavillon-Industrie-HD-7386, (CFranck-Juillot les principales maquettes et objets ayant été présentés dans le pavillon Schneider intègrent les collections, comme le grand plan en relief des usines du Creusot en 1900 ou encore celui des usines en 1836. On y trouve aussi des collections d'armes et notamment des canons fabriqués au Creusot. À l'instar des chefs d'État, Eugène Schneider fait de son musée un lieu à la gloire du Creusot, de ses usines et de sa famille. Tous les cadeaux que reçoit le maître de forges y sont déposés. Et quand ils revêtent un caractère diplomatique important, ils sont mis en vitrine, comme ce fut le cas pour des poupées japonaises.

bombardements du Creusot, la reconstruction, le changement de gérance à la tête de la société Schneider font que les collections sont oubliées. En 1953, l'idée de reconstituer un musée est de nouveau d'actualité. Une sorte de ce que nous appellerions aujourd'hui un pré-programme est réalisée dans lequel les objets disponibles pour l'exposition sont décrits, accompagnés de leur état. Parmi les maquettes aujourd'hui disparues, se trouvaient celles du pont Alexandre III sur la Seine et du pont du Danube à Vienne; le plan en relief des usines du Creusot de 1878, ou celui du quartier de la gare du Creusot. Le Wagon-Salon personnel d'Eugène Schneider enfin, a été détruit en $1963^{4}$.

30 Le nouveau musée Schneider ne voit pas le jour immédiatement. Il faut attendre la mort de Charles, le dernier membre de la dynastie des maîtres de forges, pour qu'il soit reconstitué. Installé dans la salle du Jeu de Paume du Château de la Verrerie, il est plus particulièrement consacré à l'histoire industrielle du Creusot. Comme le précédent et contrairement aux musées industriels créés à partir de la fin du XIXe siècle dans différentes villes françaises (Cartier 2004), le musée n'a pas vocation à être ouvert au public. Il est réservé à quelques invités et aux élèves des écoles Schneider ${ }^{5}$. Outre les 
collections historiques, ils peuvent y découvrir des maquettes réalisées par leurs aînés dans le cadre des écoles d'apprentissage, comme la reproduction de la machine à vapeur de 150 chevaux installée en 1892 dans la première centrale électrique des mines de Decize, à La Machine ${ }^{6}$. Cette maquette a été réalisée par les apprentis de l'année scolaire 1934-1935.

31 À sa création, en 1985, l'Académie François Bourdon hérite de ces collections, ou du moins des pièces qui ont résisté à l'épreuve du temps. Elles forment un ensemble d'environ un millier d'objets qui vont des médailles reçues par les Schneider aux maquettes de production, halles industrielles, ponts ou encore locomotives, bateaux, machines à vapeur mobiles et fixes ; on compte aussi des peintures, des statues et bien évidemment les bustes des Maîtres de forges. Enfin, les deux pièces maîtresses sont incontestablement les grands plans en relief déjà évoqués. Ils représentent, pour le premier, l'usine du Creusot en 1836 et, pour le second, l'usine en 1900. Ils ont été réalisés afin d'être exposés en 1900 dans le pavillon de la société Schneider et Cie lors de l'exposition universelle de Paris.

En 1995, en s'appuyant sur cette collection, l'Académie François Bourdon a réalisé, dans la salle du jeu de Paume du Château de la Verrerie, une première exposition permanente intitulée «Le Métal, la Machine et les Hommes" (1997). Cette dernière vient en complément de l'exposition présentée la même année par l'Écomusée de la Communauté Urbaine Le Creusot-Montceau (Corrias, Le Foll, Moëllo 2020) associée au musée d'Orsay et intitulée « Les Schneider, Le Creusot. Une famille, une entreprise, une ville (1836-1960) » (1995). En 2012, l'exposition " Le Métal, la Machine et les Hommes » arrivant à son terme, s'est posée la question de son remplacement et, avec elle, la double problématique de la mise en scène de l'histoire industrielle du Creusot de concert avec les activités actuelles.

33 Au cours des dernières années, l'ancienne exposition avait mis en lumière le manque cruel de discours sur l'industrie contemporaine. Le visiteur quittait les lieux avec le sentiment erroné que l'industrie avait disparu du Creusot. La nouvelle exposition devait casser cette représentation et permettre de comprendre le dynamisme économique creusotin, d'où l'interrogation quant à la mise en scène de l'industrie. Comment montrer, sans négliger les collections anciennes, l'industrie actuelle? Comment faire comprendre que l'industrie creusotine est une " grosse » industrie sans être une industrie grossière, mais au contraire une industrie de très haute précision ?

Le discours envers les jeunes est aussi un sujet important. Un des leitmotivs de l'histoire industrielle, c'est la question de la main d'œuvre. L'industrie était demandeuse d'ouvriers en grand nombre. Elle a maintenant besoin de disposer d'une main d'œuvre qualifiée, voire très qualifiée. Paradoxalement, dans un contexte de chômage de masse, un déficit de jeunes formés à ces métiers est perceptible. Comment sensibiliser la jeunesse aux formations qui débouchent sur des emplois dans l'industrie ? Comment parvenir à casser la représentation genrée de ces métiers et faire en sorte que les jeunes filles s'engagent aussi dans ces formations? Enfin, la dernière problématique, qui n'est pas la moindre, concerne la mise en scène des collections et leur présentation. Comment donner aux visiteurs du plaisir lors d'une visite sur des thématiques liées à la technique et à l'industrie ? Comment susciter des émotions du même ordre que celles que l'on éprouve devant une magnifique œuvre d'art? 
Fig.4. Quand l'industrie rejoint l'art...

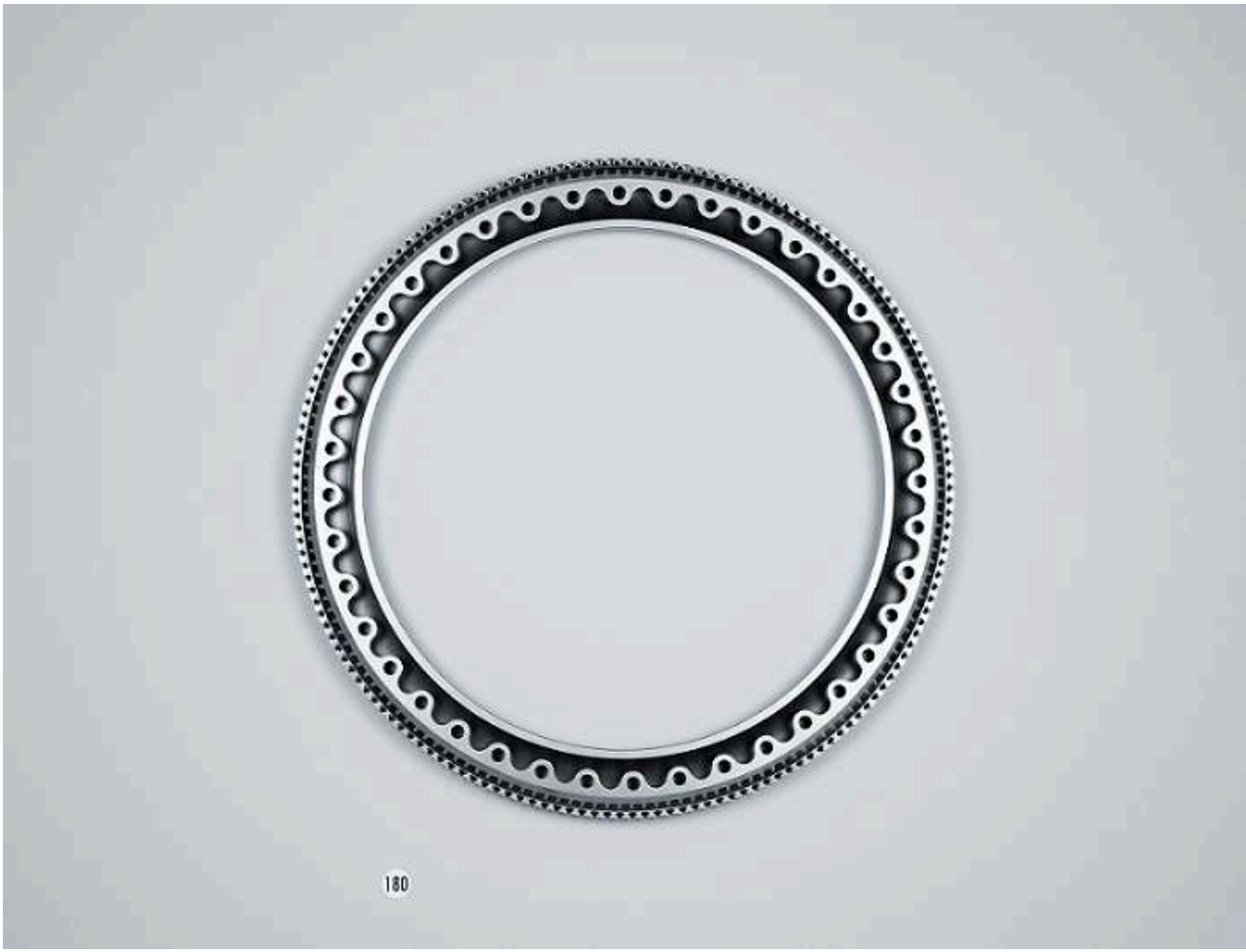

Disque de turbine basse pression de réacteur d'avion, type CFM 56, fabriqué par le groupe Safran. Pavillon-Industrie-HD-7545, (c) Franck-Juillot

\section{Montrer et donner à comprendre l'industrie, d'hier à aujourd'hui : le centre d'interprétation}

L'objectif du Pavillon de l'Industrie est certes de montrer l'industrie mais surtout de la donner à comprendre d'hier à aujourd'hui. La notion « d'aujourd'hui » est importante. La représentation que le public se fait de l'économie creusotine ne correspond pas à sa vitalité. Au mieux, il n'a aucune représentation; il ne sait pas qu'il existe des industries au Creusot. Au pire, ils pensent qu'elles ont disparu lors de la crise du milieu de la décennie 1980, suite à la faillite du groupe Creusot-Loire. 


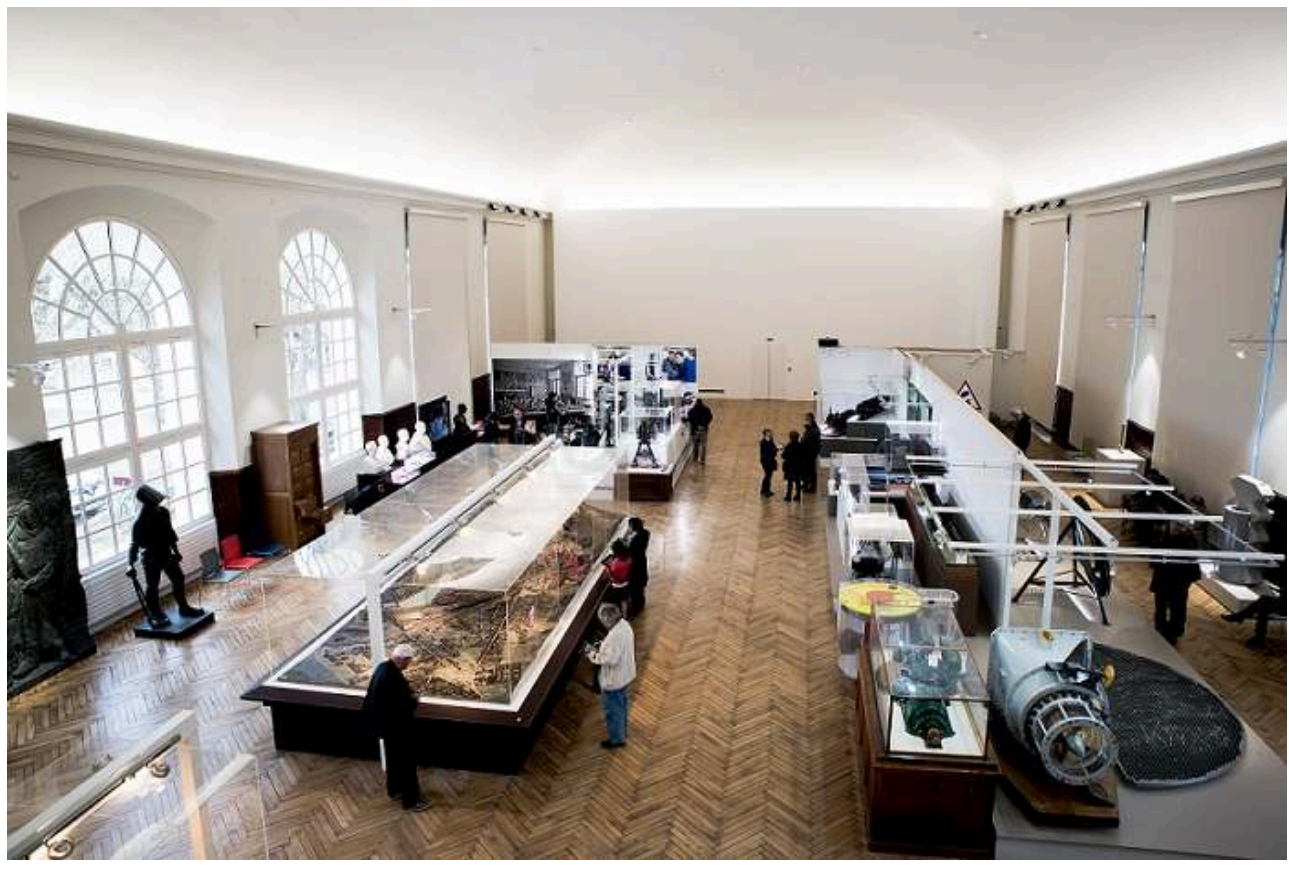

Le Pavillon de l'industrie constitue l'un des sites patrimoniaux du Château de la Verrerie, au Creusot. Conçu comme un centre d'interprétation, il propose de faire comprendre comment les industries se sont développées et interagissent avec le développement de la ville, à la fois sur le plan social et urbanistique. https://www.pavillon-industrie.fr/chateau-de-verrerie

Pavillon-Industrie-HD-7545, (CFranck-Juillot

Au-delà de ce premier message qui veut montrer que Le Creusot est un exemple réussi d'une continuité industrielle, le Pavillon de l'Industrie porte deux autres slogans forts, «l'industrie a sa place dans le paysage économique français » et «il y a des places à prendre pour les jeunes dans les métiers industriels». Ce discours est constitutif du Pavillon de l'Industrie et, en cela, il fait de ce lieu un centre d'interprétation, où la communication au public prime sur les collections. Comme centre d'interprétation, le Pavillon de l'industrie ne résulte pas de la démarche de constitution d'un patrimoine. S'impose d'abord ce que l'on veut dire et communiquer au public (Chaumier et Jacobi 2009 : 10). Le visiteur est au centre de la démarche. Pour lui donner à comprendre l'industrie dans toutes ses dimensions, le choix a été fait de s'appuyer sur une tablette interactive et sur une collection variée d'objets patrimoniaux et d'objets caractéristiques des productions industrielles actuelles.

La conception du Pavillon de l'Industrie a suivi la démarche classique pour ce type de réalisation, en commençant par la création d'un projet scientifique et culturel, la mise en place des grands messages, la programmation des collections ${ }^{7}$, puis leur mise en scène par une architecte scénographe. Ces derniers points ont nécessité un double travail avec deux prestataires différents. La mise en scène des objets réels a été réalisée par une architecte scénographe, mais, comme le choix a été fait d'utiliser une tablette numérique comme outil de médiation, il a fallu travailler la programmation des collections virtuelles présentes dans la tablette ainsi que leur scénographie. 


\section{Séquence 1. L'histoire d'un grand site industriel} aujourd'hui, le parcours repose sur des objets historiques, issus des collections de l'Académie François Bourdon et des objets contemporains mis à dispositions par les industriels partenaires. Ce parcours est divisé en 5 séquences. La première s'appuie sur les deux plans en reliefs ${ }^{8}$. Pièces maîtresses du Pavillon de l'Industrie, ils sont placés à proximité l'un de l'autre, afin de permettre aux visiteurs de voir l'extension considérable du site industriel sous la gestion de la société Schneider et Cie.

Fig.6. Détail du plan en relief de l'usine du Creusot en 1900

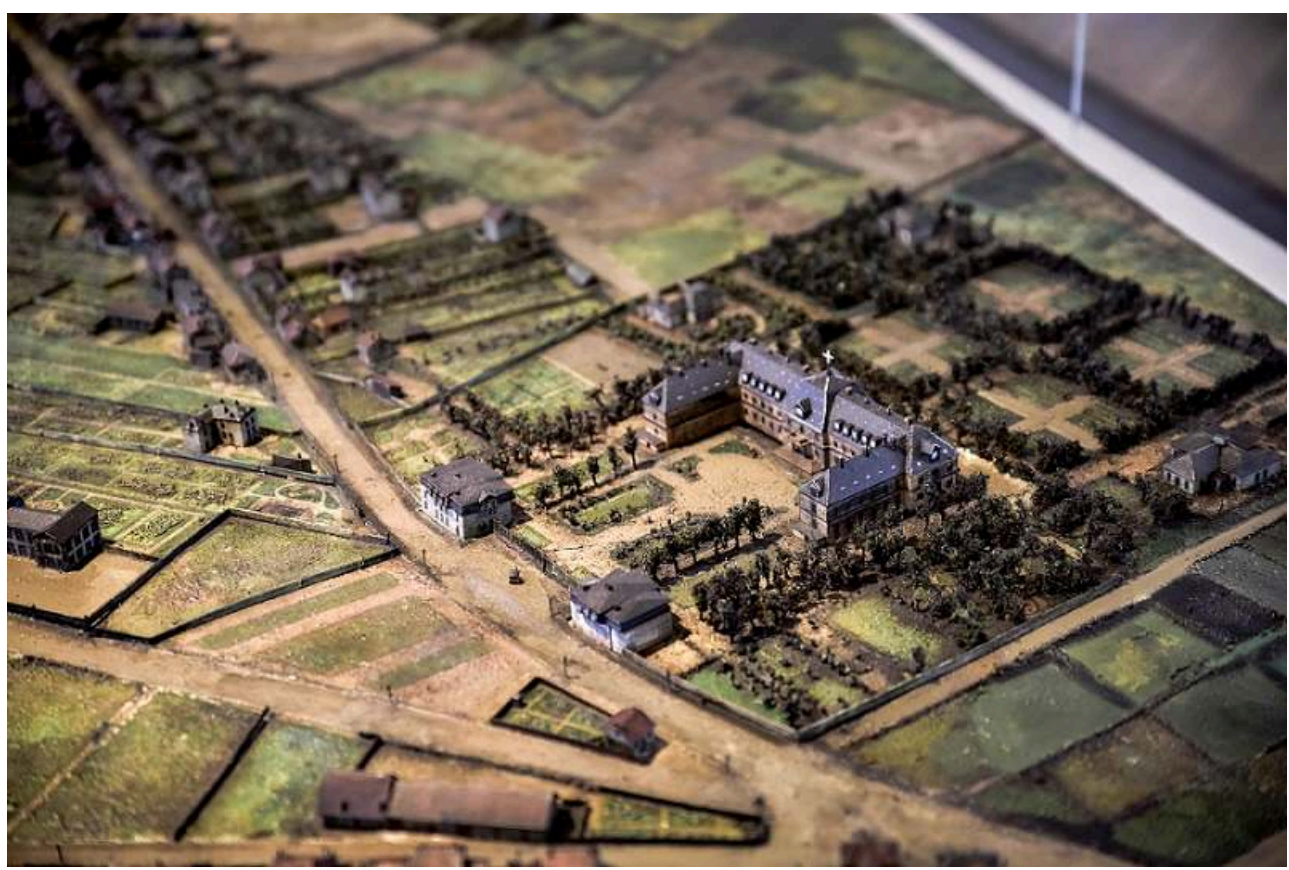

Pavillon-Industrie-HD-7259, (CFranck-Juillot

Le premier plan en relief, représentant l'usine du Creusot en 1836, permet de développer un discours sur les conditions de la création de la fonderie de fonte en 1782 par François-Ignace de Wendel. Dans ce contexte est mise en avant la haute technicité de cette entreprise qui utilise, dès son origine, des procédés de pointe importés de Grande-Bretagne, comme le coke, le haut-fourneau au coke et la machine à vapeur de James Watt. Autour du second plan en relief, plusieurs discours sont développés. La première intervention porte sur l'extension de l'usine du Creusot depuis son rachat par les frères Adolphe et Eugène Schneider ${ }^{9}$. Le plan en relief permet de montrer la transformation radicale de la plaine des Riaux, où était implantée l'ancienne fonderie.

À partir de 1860, l'usine déborde de son berceau d'origine, pour s'agrandir vers le sudest. On y découvre le bâtiment de la grande forge, celui des presses et pilons ainsi que les ateliers d'artillerie nord et sud. Le développement de l'usine depuis son rachat par les frères Schneider témoigne de la puissance industrielle du Creusot dans le dernier tiers du XIX ${ }^{\mathrm{e}}$ siècle. Le site devient le symbole industriel de la France et « la plus grande usine d'Europe » pour reprendre le titre du chapitre qui lui est consacré dans le manuel scolaire « Le tour de France par deux enfants» (Bruno $1877: 109-117)^{10}$. 


\section{Séquence 2. La question sociale et l'urbanisation}

Au-delà d'un discours historique, le plan en relief permet aussi de matérialiser une partie de l'industrie d'aujourd'hui quand elle occupe les bâtiments d'hier. C'est le cas de l'ensemble des halles industrielles construites à partir de 1860 qui hébergent les établissements d'Industeel France, de Framatome, de Thermodyn, GE Baker Hughes et d'Alstom transport ${ }^{11}$. Dans le parcours proposé aux visiteurs, l'état actuel de l'industrie creusotine est présenté pour la première fois. Au-delà du récit, le parti a été pris de montrer des films institutionnels d'AREVA NP (aujourd'hui Framatome) et Industeel. Le grand plan en relief de l'usine du Creusot montre les spécificités de l'urbanisation. L'usine occupe le centre pendant que les quartiers se développent autour. La présence sur ce plan de logements, d'une cité ouvrière, d'écoles et de l'hôtel-Dieu permet de faire la transition avec la partie suivante, "des femmes et des hommes", consacrée à la question sociale et à la présentation de la dynastie des maîtres de forges.

Fig.7. Plan type de maisons ouvrières

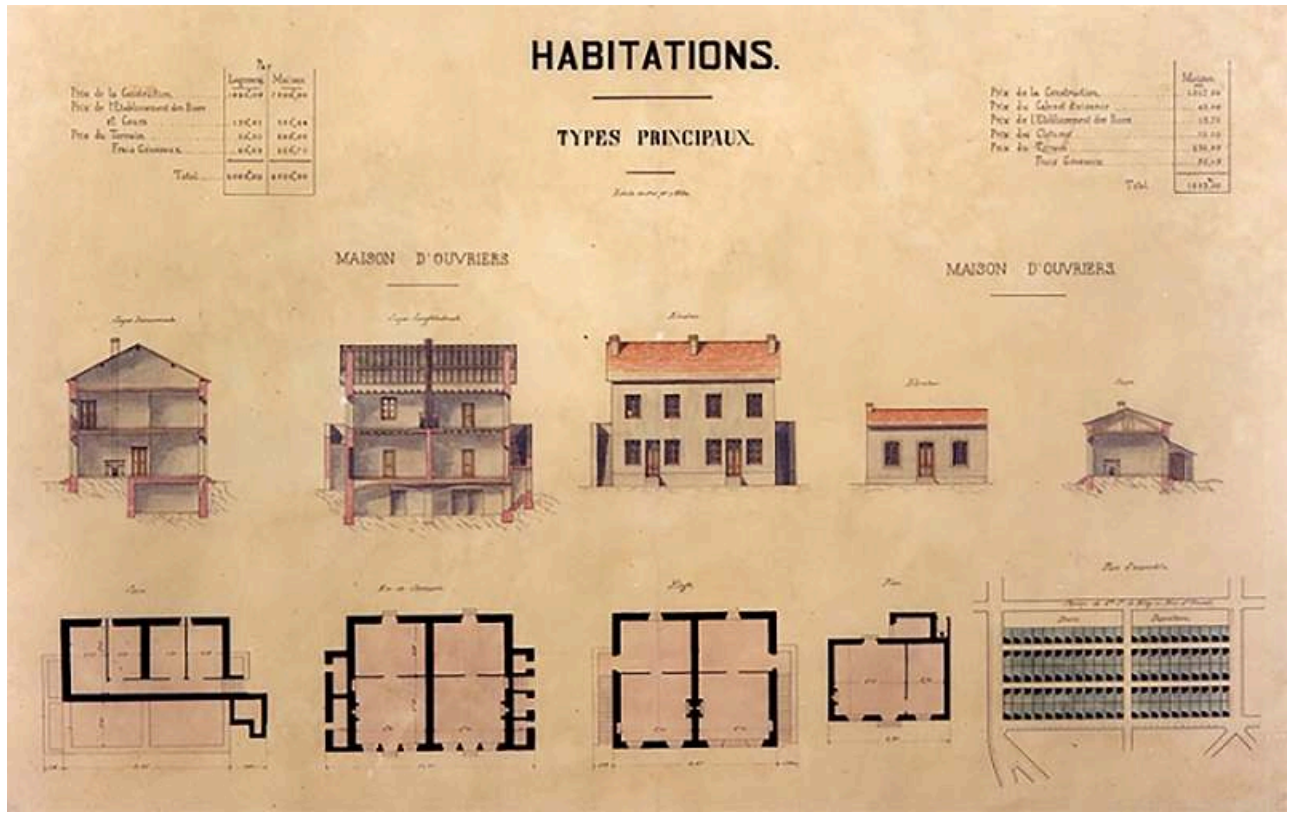

Réalisé en 1867, ce plan type présente, outre les détails architecturaux, le coût des maisons et l'agencement du « lotissement ».

Centre d'Archives de l'Académie François Bourdon, La Verrerie, Le Creusot (Saône-et-Loire)

Les visiteurs découvrent, outre les bustes des représentants de la dynastie des Schneider ${ }^{12}$, de magnifiques statues d'employés et d'ouvriers du Creusot ${ }^{13}$. Comme pour la partie relative à l'industrie, la présentation des salariés évoque à la fois les temps passés et le présent. Si la distance historique facilite le discours sur les salariés d'hier et permet de s'appuyer sur quelques anecdotes, l'écriture d'un propos sur le personnel d'aujourd'hui est un exercice plus compliqué. Le biais a été pris de l'évoquer à travers la question de la baisse des effectifs dans l'industrie qui s'accompagne d'une augmentation de la qualification des ouvriers, ainsi que de la forte diminution de la pénibilité autorisant l'emploi de femmes. 


\section{Séquence 3. La formation des ouvriers et la recherche industrielle}

La troisième séquence aborde la formation et la recherche. Autour d'objets emblématiques, le contenu porte sur la formation initiale mise en place par la société Schneider dès 1837 , et plus particulièrement sur les savoir-faire et la recherche. La dimension historique est illustrée par les objets présentés.

Fig.8. La formation scolaire aux usines Schneider au début du XXe siècle

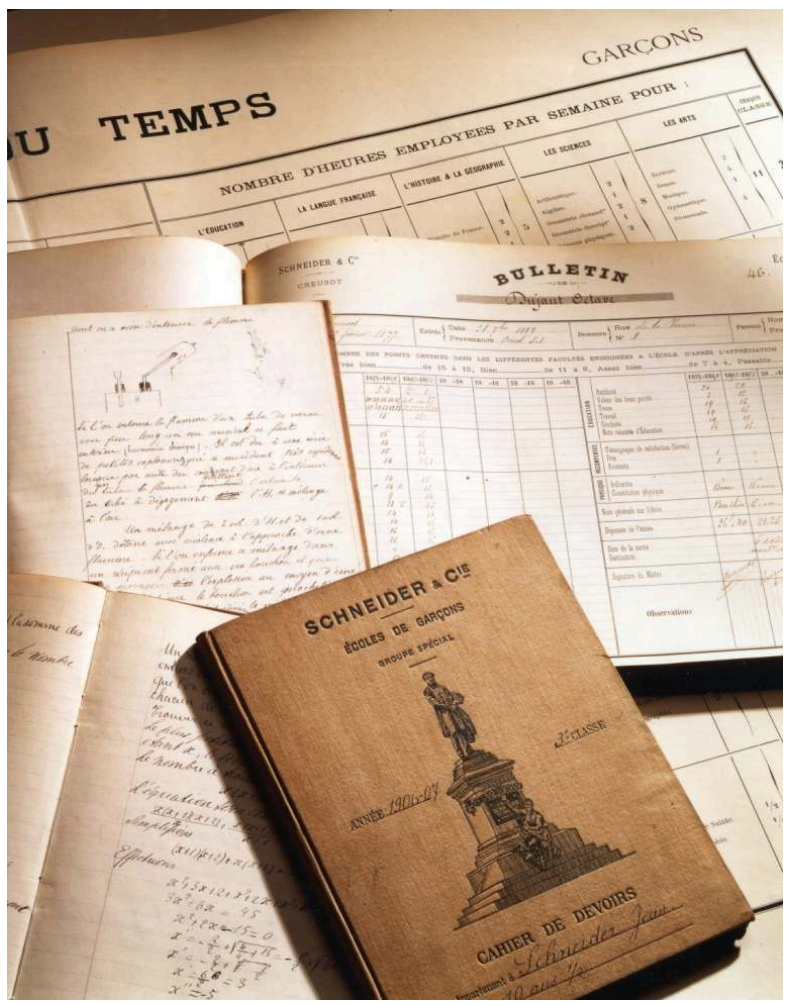

Emploi du temps, cahiers de devoir, bulletin scolaire... Prise très au sérieux, la formation scolaire des futurs ouvriers était le garant de leur technicité et de leur qualification.

Centre d'archives de l'Académie François Bourdon, La Verrerie, Le Creusot (Saône-et-Loire)

Une place particulière est attribuée à un uniforme d'écolier Schneider. Des supports pédagogiques permettent de comprendre des principes mécaniques simples; des instruments de laboratoire de recherche ou encore la maquette du marteau-pilon expérimental de François Bourdon illustrent la dimension recherche. Les reproductions réalisées par les élèves des écoles Schneider, comme celles du marteau-pilon de 100 tonnes et d'une machine à vapeur fixe de 150 chevaux, portent la question des savoirfaire. 
Fig.9. Maquette du marteau-pilon à vapeur de 100 tonnes

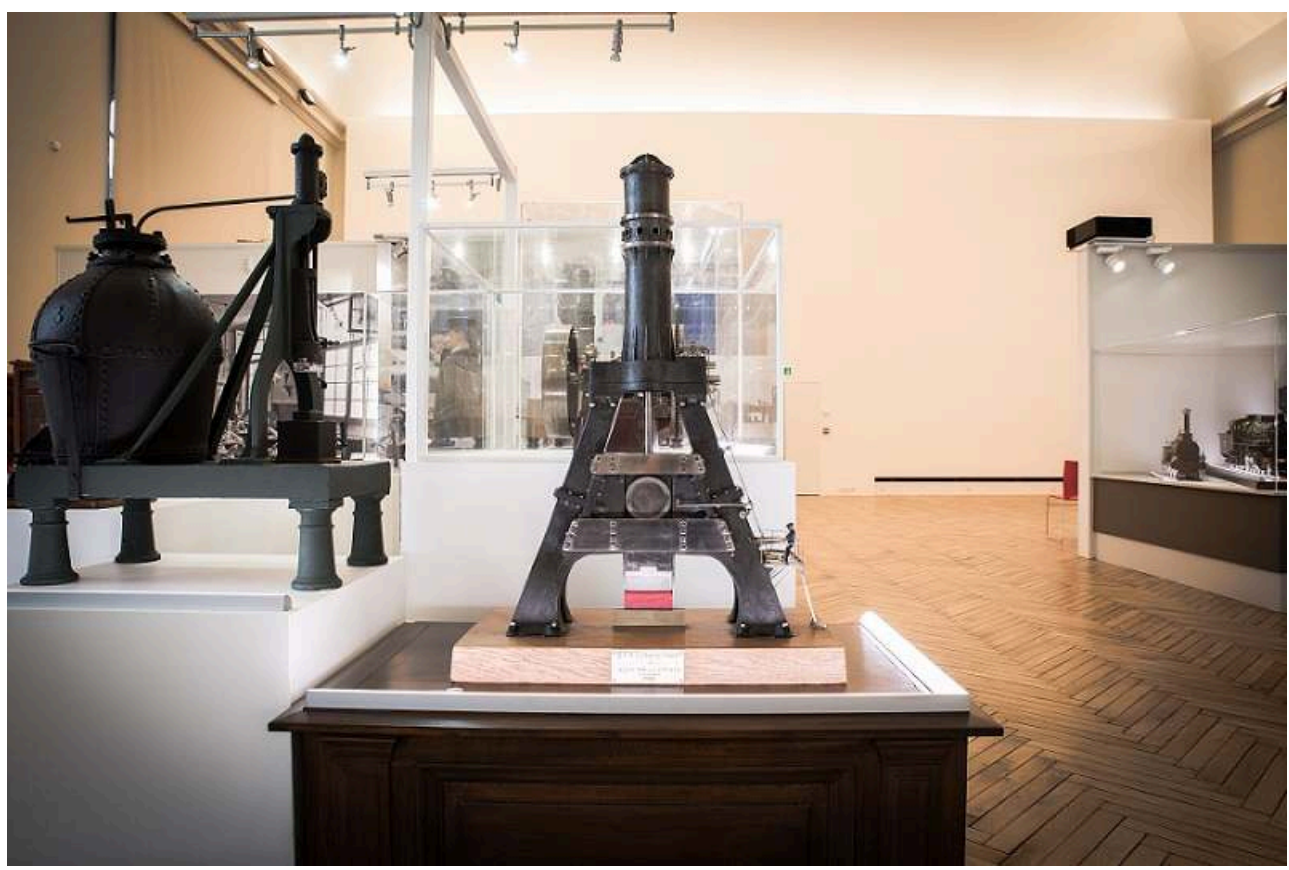

Maquette au 1/20e réalisé par les apprentis de l'école Schneider en 1955.

Pavillon-Industrie-HD-7350, (CFranck-Juillot

Il en va de même du magnifique vase à pampres réalisé par Honoré Balzon vers 1890. La spécificité de ce vase entouré d'une guirlande de feuilles est d'avoir été réalisé sans soudure, simplement par étirement d'une seule pièce de métal. La partie contemporaine, liée à la recherche et à la formation, est traitée uniquement par une approche numérique, sur la tablette, à travers le témoignage de la directrice du Centre de recherche sur les matériaux du Creusot et celui d'un professeur de l'université de Dijon. Il explique le futur de la métallurgie des poudres. Pour la formation et les métiers de l'industrie, plusieurs vidéos présentent, à travers des témoignages de jeunes embauchés, quelques-unes des filières.

Enfin, par le wifi, le visiteur a accès, toujours via la tablette numérique, à la base de données du site metalemploi.org qui permet de découvrir en temps réel les offres d'emploi dans les métiers de la métallurgie et des constructions mécaniques disponibles en France. Le seul rappel qui est fait dans la scénographie physique du Pavillon de l'Industrie du temps présent, c'est une immense photographie, prise en 2015, de jeunes étudiants au travail à l'IUT du Creusot. Ce document est placé en regard d'une photographie d'une classe d'élèves d'une école Schneider prise au début du XX siècle.

\section{Séquence 4. De l'acier aux machines, les produits de la grande industrie}

47 La dernière séquence, intitulée "De l'acier aux machines", est consacrée aux productions. Elle est traitée selon le même principe, c'est-à-dire d'hier et d'aujourd'hui. Les productions d'hier sont mises en avant à travers des objets, maquettes et tableaux issus des collections de l'Académie François Bourdon. Les productions actuelles sont illustrées par des maquettes et objets mis à notre disposition par des partenaires 
industriels. Cette dernière partie constitue l'élément emblématique de la scénographie physique du Pavillon de l'Industrie. Des exemples de productions actuelles issues des industries du territoire sont présentés. Le visiteur peut découvrir une demi-plaque entretoise d'un générateur de vapeur d'une centrale EPR, un rotor de compresseur centrifuge, un échantillon de tôle plaquée d'acier inoxydable sur base d'acier au carbone ou encore un longeron de boggie de RER. Toutes ces productions sont issues des établissements creusotins des groupes Framatome, GE Baker Hughes, IndusteelFrance ou encore Safran.

Fig.10. Détail d'une demi-plaque entretoise d'un générateur de vapeur

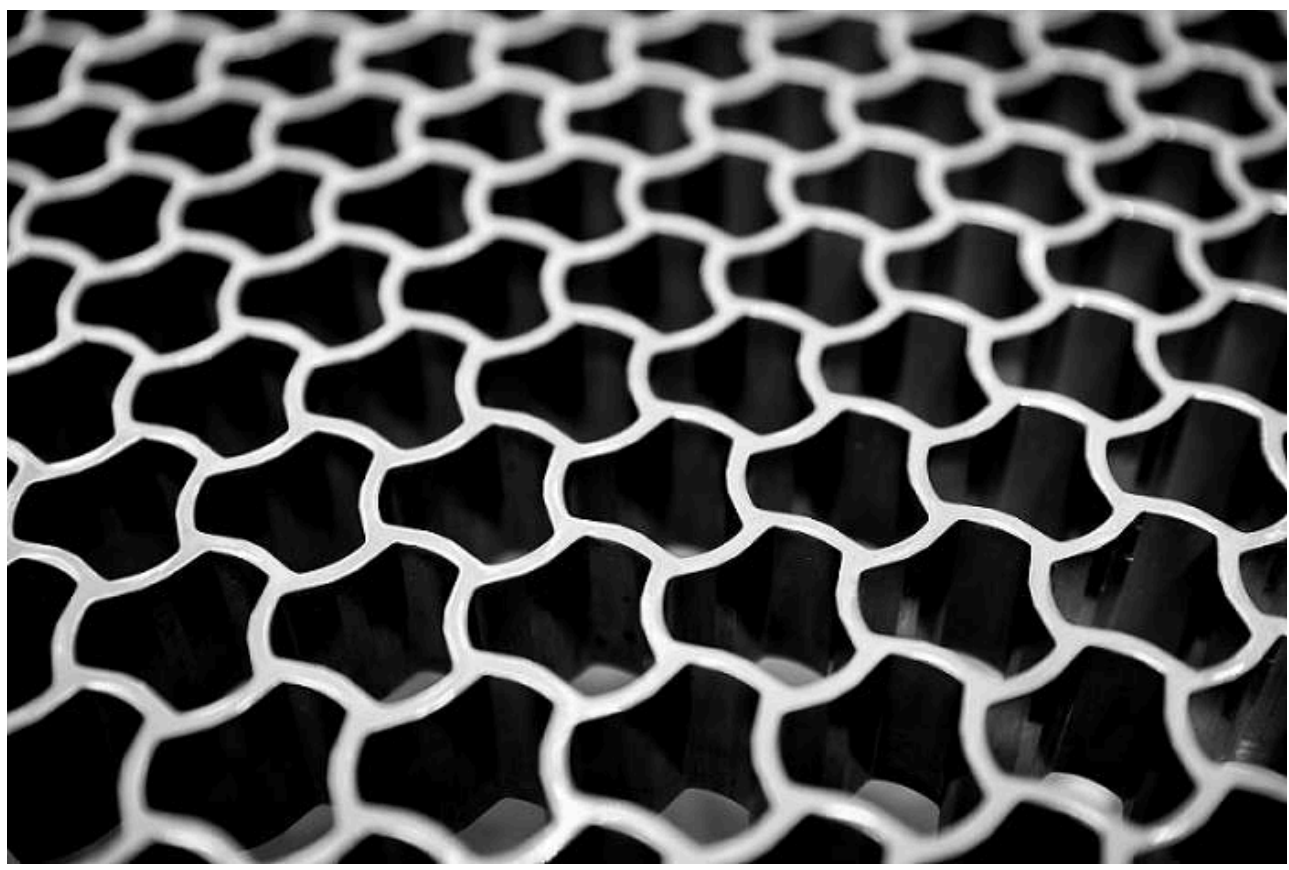

Générateur de vapeur pour réacteur EPR produit par Framatome.

Pavillon-Industrie-HD-7268, (CFranck-Juillot

\section{Séquence 5. Les Petites et Moyennes Industries du territoire}

48 La dernière partie de la scénographie est consacrée aux productions des Petites et Moyennes Industries du territoire. Sont présentées la chaise A de Tolix, fabriquée à Autun, ou encore une aube de turbine à gaz produite par Turbine Casting. Toujours avec la volonté de donner à voir l'industrie d'aujourd'hui, la visite est ponctuée par la projection d'un film sur grand écran. Film de commande, il offre un beau moment d'émotion autour d'images mettant en avant des femmes et des hommes au travail au sein des différents établissements industriels du Creusot. Il n'y a aucun commentaire ; seuls les lieux où sont réalisées les séquences sont légendés. Lorsque l'industrie est bruyante, l'ambiance sonore est restituée; quand ce n'est pas le cas, une musique dynamique accompagne les images.

49 La mise en scène des collections réelles a été imaginée avec une volonté de casser le côté obscur de l'industrie et des techniques. Obscur, dans le sens peu accessible à la majorité des publics, non pas par la réalité des faits industriels et techniques, mais par la représentation que la plupart de nos contemporains s'en font. Pour ce faire, la 
scénographie a été réalisée avec une volonté de «mise en lumière » des collections et des espaces. Des tons clairs ont été privilégiés, accompagnés d'un éclairage chaleureux.

Dans le Pavillon de l'Industrie, au-delà des collections, ce qui compte, ce sont aussi les discours, les messages à faire passer aux visiteurs. C'est pourquoi une tablette numérique sert de vecteur auprès des visiteurs.

\section{La tablette numérique comme outil de médiation}

51 L'usage de l'audio-guide et du visio-guide n'est pas nouveau. Bon nombre de musées ou sites patrimoniaux utilisent ces outils de médiation. La tablette interactive est beaucoup moins répandue. Elle est remise au visiteur à son arrivée et son utilisation est comprise dans le billet d'entrée. Cette solution s'est imposée pour plusieurs raisons. La première est le discours. Doté d'un casque, le visiteur écoute un propos dynamique, porté par deux comédiens, une jeune femme et un jeune homme. Afin de casser les représentations genrées sur les métiers de l'industrie, la jeune femme porte le contenu technique. Elle explique aux visiteurs les dimensions techniques et industrielles à travers un discours didactique. Le jeune homme porte le contenu historique qui est, lui aussi, très pédagogique. L'enjeu réside dans la vulgarisation de faits qui peuvent être complexes et ceci pour permettre aux visiteurs de passer un moment agréable tout en apprenant quelques notions simples sur l'industrie sidérurgique et celle des constructions mécaniques. Pour ce faire, le discours des comédiens est agrémenté de films représentant des scènes techniques de fonctionnement de four à coke, de fabrication de la fonte, de l'acier et de diverses productions.

Des photographies accompagnent les discours, notamment ceux sur la dimension urbanistique et sociale avec des vues de cités ouvrières au XIX ${ }^{\mathrm{e}}$ siècle, des écoles, des soins, les trois piliers de ce que l'on appelle communément le paternalisme. Des documents d'archives apportent un complément. Des animations de synthèse très didactiques offrent la possibilité de comprendre quelques notions techniques, comme la fabrication de la fonte dans un haut-fourneau, le fonctionnement d'une machine à vapeur alternative, d'une turbine à vapeur, d'un compresseur centrifuge ou encore d'une centrale nucléaire. C'est la grande valeur ajoutée de la tablette qui apporte une bonne visualisation grâce à son écran de 10 pouces. La dimension tactile de la tablette, outre la navigation, permet aussi quelques jeux qui agrémentent la visite et lui confèrent une dimension ludique.

La tablette numérique entend aussi manifester la volonté d'illustrer la modernité de l'industrie. L'image de la tablette est positive, à l'instar de l'industrie du Creusot. C'est encore un outil peu fréquent que le public n'a pas l'habitude de voir dans un tel lieu. De surcroît, la tablette retenue est l'une des rares assemblées en France et devinez où ?... $\mathrm{Au}$ Creusot !

Outil de médiation, la tablette comprend l'ensemble des contenus scientifiques du centre d'interprétation, y compris les cartels des objets. La relation entre les objets réels et les cartels passe par un numéro apposé à proximité des objets qui renvoie au cartel présenté dans la tablette. C'est un choix important qui a été adopté afin de ne pas poser de cartels à proximité des objets. L'objectif est d'inciter le visiteur à utiliser la tablette. C'est elle qui lui permet de comprendre les différentes séquences évoquées 
plus haut. Bien évidemment, elle lui sert à se repérer et à se déplacer au sein du Pavillon de l'Industrie.

Au même titre que la mise en scène des objets, les contenus de la tablette ont bénéficié d'une véritable scénographie. Là encore, le maître-mot est de placer en regard le passé et le présent de l'industrie. Chaque séquence est illustrée par un cliché ancien suivi d'une photographie contemporaine. Le discours porté par les deux comédiens est entrecoupé d'animations (films, photographies, animations virtuelles, jeux, etc.) que le visiteur peut interrompre à sa guise. La capacité de mémoire de la tablette autorise un contenu très riche et volumineux, ce qui offre des perspectives d'enrichissement. La limite ne se situe pas dans les volumes, mais plutôt dans la capacité actuelle du système Androïd. Ce dernier est moins souple qu'un système sur PC et ne permet pas d'exécuter toutes les volontés d'un commissaire enthousiaste de l'usage de cet outil !

Le visiteur a le choix de suivre la visite complète du Pavillon de l'Industrie avec un contenu interactif. Cette visite prend à peu près 2 heures. Il dispose aussi de la possibilité de choisir l'option « $45 \mathrm{mn}$ », prise en charge par la tablette, au même titre qu'avec un guide. Dans ce cas, il ne bénéficie plus de l'interactivité. Le contenu est transformé en un film qui correspond à une visite rapide. La dernière option est celle des seuls cartels. Le visiteur ne dispose que des cartels et peut donc organiser une visite comme dans un musée classique. Cette option est intéressante pour un groupe accompagné d'un guide. L'ensemble des contenus est accessible en français et en anglais.

\section{Les usages du Pavillon de l'Industrie}

Dès l'origine, le Pavillon de l'Industrie a été pensé en lien étroit avec les industriels du territoire : l'Éducation nationale ainsi que les collectivités locales. Pour sa conception puis le suivi de sa réalisation, un comité de pilotage a été mis en place. Il était formé de représentants des industriels partenaires, de la ville du Creusot et de la Communauté Urbaine Le Creusot-Montceau, du Rectorat de Dijon et de quelques personnes qualifiées. Ce comité de pilotage a joué un rôle majeur dans la conception du Pavillon de l'Industrie, dans les messages qu'il devait porter, ainsi que dans ses objectifs et ses usages futurs. Nous n'allons pas revenir sur les messages abordés plus haut. Dès sa conception, une demande forte est apparue, celle d'offrir la possibilité de réaliser dans ce lieu des manifestations comme des conférences, séminaires, repas... pour une capacité de 150 personnes. Cette demande a été portée par les industriels. Ils ont manifesté la volonté de pouvoir organiser des séminaires ou d'autres événements au sein du Pavillon de l'Industrie. Le calibrage à 150 places correspond à un besoin d'espaces intermédiaires, peu fréquent dans des lieux signifiants, contrairement aux structures pouvant accueillir plus de 150 personnes. L'architecte-scénographe a répondu à cette sollicitation en créant un espace polyvalent dans la partie dévolue à la projection du film consacré à l'industrie creusotine d'aujourd'hui.

Dans le cadre du mécénat des entreprises au profit du Pavillon de l'Industrie, il leur a été accordé la possibilité de privatiser ${ }^{14}$ le lieu, sous certaines conditions, trois fois par an et ceci pour une durée de trois années ${ }^{15}$. Les mêmes conditions ont été attribuées aux collectivités locales qui ont alloué des subventions. Dès les semaines qui ont suivi l'ouverture du Pavillon de l'Industrie, les entreprises partenaires l'ont utilisé pour réaliser différentes manifestations ${ }^{16}$. On connaît les enjeux du mécénat pour les 
entreprises, mais au-delà de " la communication par l'évènement » (Piquet et Tobelem 2006 : 57) auquel les soutiens au projet ont recours, une véritable fierté des entreprises locales se manifeste au moment de mettre en scène leurs manifestations au sein du Pavillon de l'Industrie, au cœur de collections porteuses de l'image industrielle du territoire. Il en est de même pour la ville du Creusot qui y organise régulièrement des activités, comme les populaires réunions de quartier; ou encore la Communauté Urbaine Le Creusot-Montceau, quand elle signe l'accord avec l'Université de Bourgogne, la Région Bourgogne Franche-Comté et le Pôle nucléaire de Bourgogne pour créer l'association Excalibure qui portera la recherche sur la métallurgie des poudres.

59 L'accueil des publics scolaires constitue une mission importante du Pavillon de l'Industrie. Lors de sa conception, un comité pédagogique a été mis en place en lien avec le Rectorat de Dijon. Enseignants de diverses disciplines, Inspecteurs Pédagogiques Régionaux, membres du Centre d'Information et d'Orientation (CIO) ont travaillé sur les messages à apporter aux scolaires avec le concours d'enseignants détachés auprès du service éducatif de l'Académie François Bourdon. De ce travail, il est ressorti la nécessité de profiter des capacités de la tablette numérique pour permettre aux enseignants qui souhaitent visiter le Pavillon de l'Industrie avec leur classe de disposer de modules pédagogiques. Ils peuvent les assembler à leur guise pour réaliser un parcours pédagogique. À terme, ils pourront faire travailler leurs élèves sur les matériaux (fonte, fer, aciers spéciaux, supers alliages...), l'énergie, les constructions mécaniques avec les techniques utilisées, les questions sociales... Les ressources sont gratuitement mises à disposition des enseignants via le site Internet du Pavillon de l'Industrie ${ }^{17}$. Il est aussi prévu qu'ils aient accès à l'ensemble des modules pédagogiques. D'ores et déjà, la question des métiers de l'industrie est traitée sur la tablette, à travers des fiches réalisées par le $\mathrm{CIO}$ ou encore des témoignages vidéo de jeunes recrues dans les industries partenaires. L'enjeu est important car, comme nous l'avons déjà mentionné, l'industrie a du mal à recruter. C'est en tous cas ce que démontre régulièrement l'étude réalisée par Pôle emploi «Besoin de main-d'œuvre des entreprises - $\mathrm{BMO}^{18} »$. La cause principale est bien connue: elle réside dans l'inadéquation entre le profil recherché par l'entreprise et la formation des candidats potentiels. Revaloriser auprès des jeunes les filières de l'industrie constitue aussi un des objectifs du Pavillon de l'Industrie.

60 À côté de l'écriture d'une histoire industrielle et s'appuyant sur les travaux des historiens, le centre d'interprétation permet de vulgariser auprès d'un large public le fait industriel. Ancré sur un territoire éminemment industriel, contre-exemple de la désindustrialisation qui touche la France, il est le fruit d'une tradition de mise en scène de l'industrie, comme nous pouvions la découvrir au cours des expositions universelles du XIX ${ }^{e}$ siècle. Il répond aussi à un besoin des acteurs du territoire de montrer et d'expliquer l'industrie d'aujourd'hui avec ses enjeux. Ainsi, il porte un discours positif et militant sur la nécessaire place de l'industrie dans l'économie. Il est aussi un des lieux où les plus jeunes peuvent découvrir les métiers et filières qui lui sont liés. Les choix technologiques qui ont été faits pour la médiation avec les publics, à travers l'usage de la tablette numérique, placent le Pavillon de l'Industrie dans une modernité qui illustre celle de l'industrie d'aujourd'hui et ouvre sur celle du futur. 


\section{Les archives industrielles}

61 À côté des collections d'objets, le patrimoine industriel du Creusot compte également des fonds d'archives d'une rare richesse. Ils sont aussi conservés et gérés par l'Académie François Bourdon ${ }^{19}$.

Les archives directement liées à l'histoire industrielle du Creusot ont été constituées par l'usine. Elles offrent aux chercheurs une masse de documents très diversifiée ${ }^{20}$. Cela s'explique en partie par la longue période que couvrent les fonds, de la veille de la première révolution industrielle au cadre industriel actuel. C'est aussi lié à la haute technicité des productions réalisées. En termes d'archives, les contraintes associées au maintien d'un haut niveau technique se traduisent par une masse importante de documents centrés sur les équipements industriels et les productions. Les premiers documents permettent aux chercheurs d'appréhender la constitution et le développement des différents établissements et services de la société Schneider ainsi que de ses filiales, tant pour ce qui concerne leur nature, leur importance que leur évolution technologique. Les fonds embrassent la plus large part du cycle d'existence d'un grand nombre des équipements, depuis l'étude initiale d'installation jusqu'à l'exploitation, en passant par la mise en fonctionnement, les mises au point et améliorations successives; ainsi, par exemple, pour les hauts-fourneaux: études, installations, modifications et reconstructions, mises à feu et exploitation. 
Fig.11. Établissements Schneider : photomontage d'archives anciennes

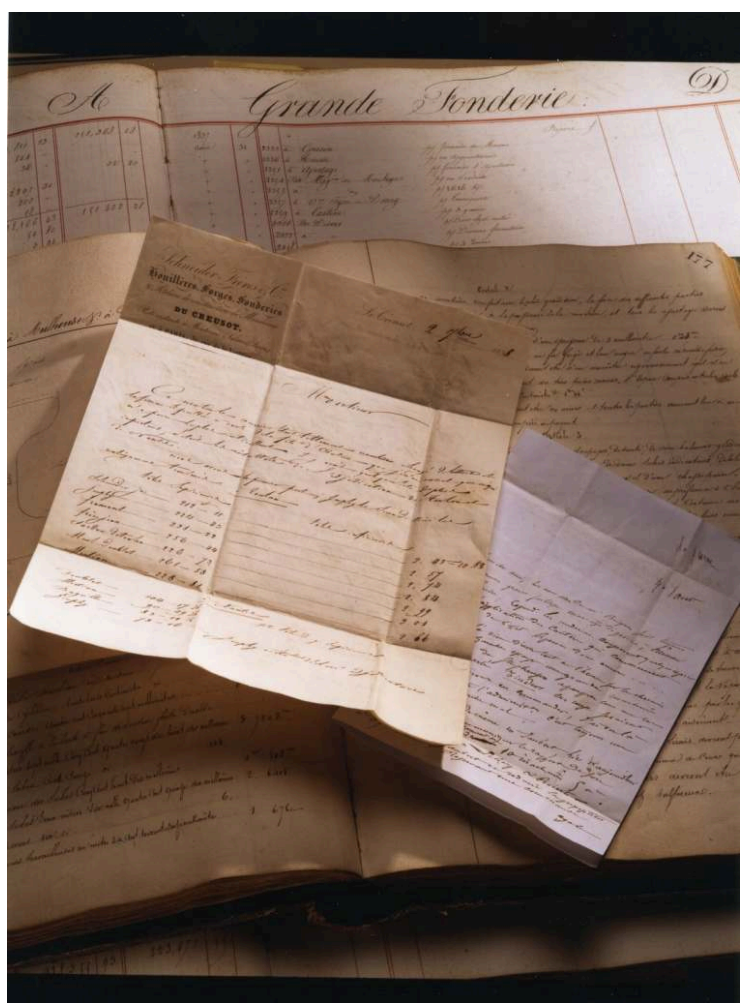

Les archives conservées à l'Académie François Bourdon représentent environ 6,5 kilomètres linéaires de dossiers, plus de 520000 documents iconographiques, 120000 plans, une bibliothèque de 50000 volumes. A cela s'ajoute une collection d'objets historiques d'environ 1200 pièces. Ce fonds constitue sans aucun doute l'un des plus grands centres d'archives industrielles du pays, avec le centre des archives du monde du travail à Roubaix, le centre historique minier de Lewarde, les fonds stéphanois, la fondation Berliet près de Lyon, les fonds patrimoniaux de St-Gobain et EDF à Blois, ceux de Mines ParisTech et de l'Institut pour l'Histoire de l'Aluminium à Clichy (92).

Centre d'archives de l'Académie François Bourdon, La Verrerie, Le Creusot (Saône-et-Loire)

63 Les dossiers sur les produits contiennent une documentation foisonnante sur les fabrications, depuis l'extraction de matières premières jusqu'aux ensembles complexes de construction mécanique ou de chaudronnerie (locomotive, machine à vapeur, moteur diesel, chaudière, etc.), et aux réalisations de travaux publics (ponts, charpentes, aménagements portuaires, etc.). Ces archives incluent aussi d'importantes informations sur les produits, l'état d'avancement du progrès technique et des procédés de fabrication d'autres sociétés, souvent concurrentes, obtenues au cours de missions conduites par des ingénieurs ou techniciens, en France comme à l'étranger.

La prise de renseignements, la veille technologique, représentent une part importante de nos fonds. Ils élargissent le champ des connaissances des différents produits et apportent un éclairage sur l'histoire de leur évolution technique. Les documents sur les produits doivent être aussi complétés par ceux des services commerciaux, avec leurs départements d'achats, de ventes, les relations avec les agences et la publicité.

Transversaux à ces thèmes, les rapports de missions sont au nombre d'environ 3 000. Ils sont conservés sous la forme des dossiers homogènes composés d'un rapport détaillé souvent accompagné de nombreuses illustrations: dessins, schémas, tableaux ou courbes. Ils couvrent une période allant de 1896 à 1957 et contiennent des informations décrivant le fonctionnement et l'organisation des entreprises ou ateliers, la gestion des 
personnels, l'organisation de leur production, les procédés de fabrication... Soulignons enfin que ces rapports de missions offrent une masse documentaire d'un grand intérêt.

Au-delà de ces documents, souvent techniques, les archives conservées par l'Académie couvrent aussi la dimension administrative des industries. Les dossiers de direction permettent de disposer d'une description globale des sociétés, de leur environnement économique et industriel (correspondance, rapports de mission...), de leur fonctionnement interne (rapports périodiques d'activités, notes aux services). Nous trouvons aussi des dossiers plus particuliers. Par exemple, certains documents traitent des expositions universelles, de la mobilisation industrielle, des nationalisations des industries de guerre (1936-1942), ou, enfin, des choix stratégiques au travers des archives produites par les instances de direction (comptes rendus de réunions des conseils ou comités). Les dossiers de direction offrent la possibilité d'étudier le système de management comme l'organisation du travail d'une grande entreprise, les relations extérieures avec la concurrence en France et dans le monde, ainsi que la politique d'expansion et de filialisation, etc.

Fig.12. Entreprise Schneider : fiche d'ouvrier de Deng Xiaoping, en 1923 (futur Secrétaire générale du Parti Communiste chinois)

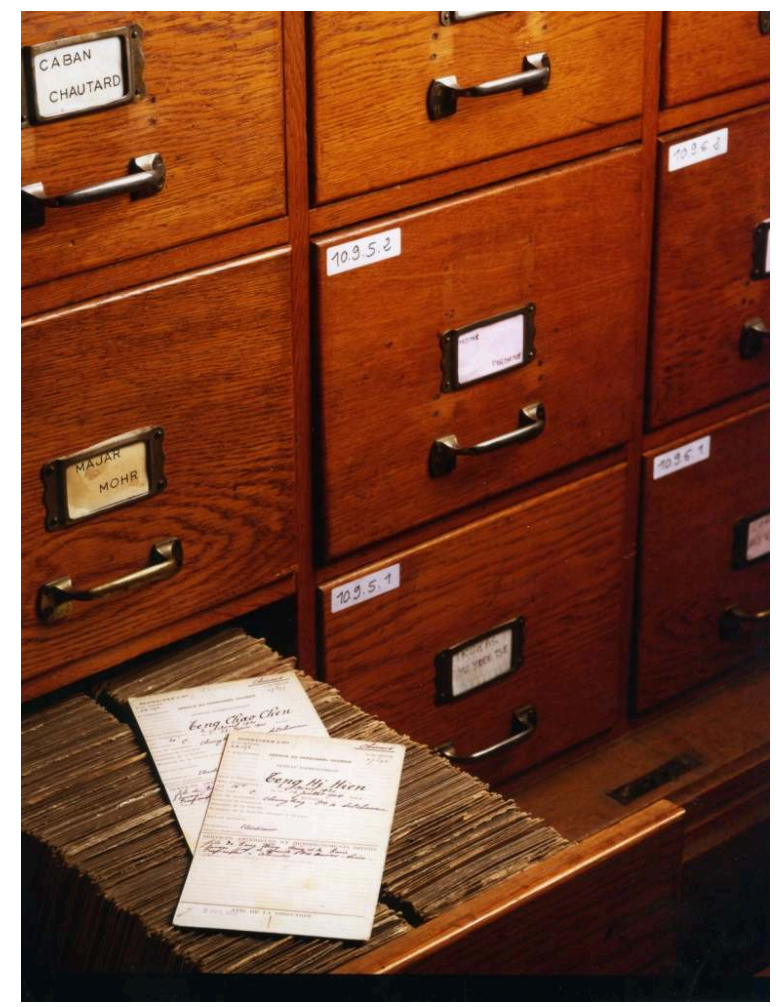

Les dossiers de direction offrent la possibilité d'étudier le système de management comme l'organisation du travail d'une grande entreprise.

Centre d'archives de l'Académie François Bourdon, La Verrerie, Le Creusot (Saône-et-Loire)

Compléments indispensables à ces dossiers de direction, en amont, le chercheur accède aux documents constitutifs des sociétés et de leur conseil d'administration. En aval, il trouve les archives des services financiers, de la comptabilité et des services du contentieux. Le chercheur peut donc entreprendre des travaux sur l'évolution du capital de l'entreprise, de sa politique d'investissements, ainsi que des études financières. Les archives de la comptabilité renferment les documents souvent 
indispensables à des études d'ensemble telle que la stratégie industrielle et financière. Les dossiers juridiques comprennent aussi ceux du contentieux, ainsi que de la propriété industrielle. Nous y trouvons également la collection des actes notariés Schneider (1836-1966), des contrats de Creusot-Loire (industriels et commerciaux), ainsi que des dossiers de procédures judiciaires et/ou administratives.

Souvent accessoires dans les industries récentes, les archives du domaine occupent une place prépondérante. Historiquement, cela s'explique par l'emprise géographique de la Société Schneider qui, outre la nécessité de se doter des infrastructures industrielles classiques, comme les bâtiments industriels, a pris en charge l'aménagement de tous les réseaux d'adduction d'éléments extérieurs (eau, électricité, gaz) et de communication (routes, voies d'eau, chemin de fer...) nécessaires à son alimentation comme au transport de ses productions et ce, dès 1836. De plus, la stratégie industrielle de concentration verticale pousse à l'acquisition de mines et carrières (Passaqui 2006, 2009), dont l'AFB conserve les plans. Enfin, ces archives renferment des éléments sur un grand nombre de bâtiments ordinaires construits par l'entreprise: écoles, cités ouvrières, maisons d'ingénieurs, bâtiments religieux, hôpitaux...

Par ce dernier point, les documents du domaine sont à rapprocher de ceux de la gestion $\mathrm{du}$ personnel et des affaires sociales. Au-delà des archives classiques du personnel, ces séries rassemblent les documents se rapportant au paternalisme "schneidérien " à travers l'ensemble des institutions qui accompagnent le quotidien des Creusotins: santé et protection sociale (Hôtel-Dieu, pouponnières, maisons de retraite, orphelinat...), éducation (écoles Schneider, formation professionnelle, écoles ménagères...), caisses de prévoyance, de secours et de retraite, ou encore loisirs (associations, centres de vacances...).

70 Outre les documents issus de l'activité industrielle des sociétés, l'Académie conserve des archives familiales des Schneider. Le statut légal de la Société Schneider et Cie, la commandite par actions, leur conférait, en tant que gérants, un rôle et un pouvoir particulièrement importants, qu'ils ont personnellement exercés. La gestion et l'archivage des documents issus de cette fonction étaient du ressort du «secrétariat particulier ", auquel les Schneider ont confié également le suivi de documents privés et familiaux. De fait, on constate qu'il n'y a pas de frontière nette entre les archives issues de la gérance et celles relatives à la famille et à la vie privée. 
Fig.13. Les loisirs et la vie culturelle des ouvriers et employés au Creusot au début du XXe siècle

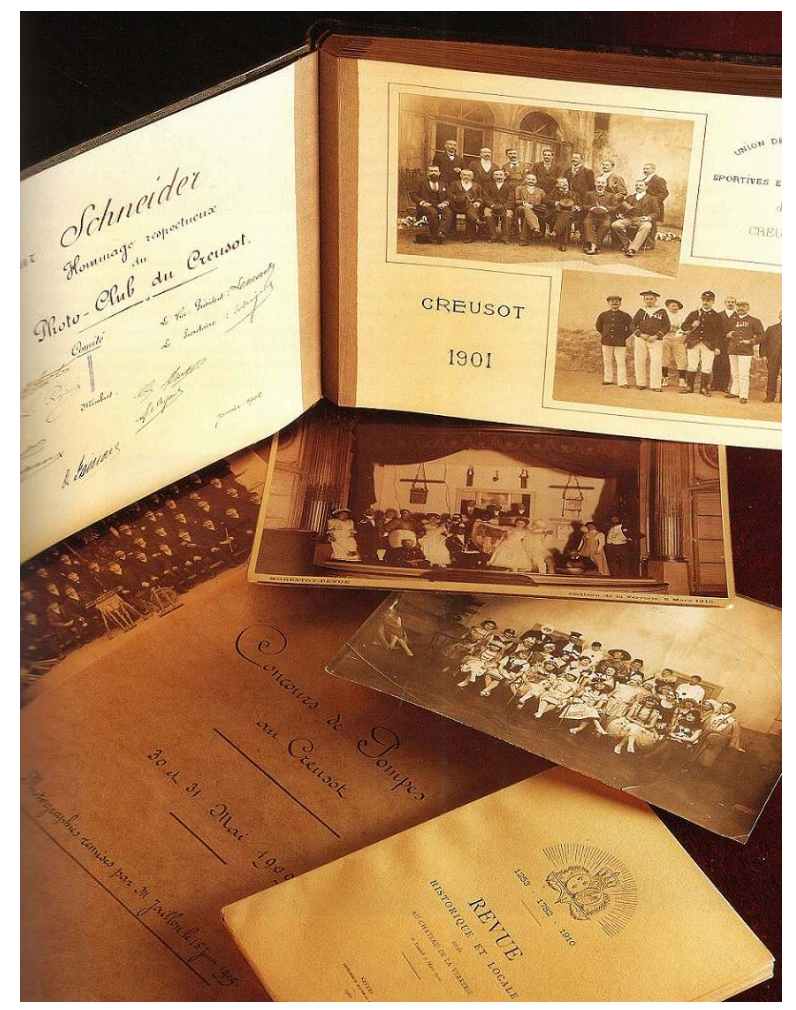

Club photo, théâtre, orchestre ( harmonie municipale »), concours de pompes, revue historique et locale : ce photomontage donne à voir ce qu'apprennent les archives Schneider de la vie culturelle au Creusot, à la Belle-époque.

Centre d'archives de l'Académie François Bourdon, La Verrerie, Le Creusot (Saône-et-Loire)

71 Ces documents sont d'un immense intérêt, notamment les correspondances privées, nombreuses dans cette série. Pour compléter, n'oublions pas le fonds «plans» et le fonds « iconographies». Le premier, composé d'environ 100000 pièces, donne un aperçu de l'évolution de l'aménagement urbain et industriel autour des sites d'exploitation, grâce notamment aux plans cadastraux, de maisons et d'édifices publics, de bâtiments industriels et d'ateliers... À travers les plans, l'évolution des techniques est perceptible pour des productions comme les locomotives à vapeur, diesels et électriques, les machines à vapeur fixes, les turbines à vapeur, les constructions navales, les moteurs, l'armement, les équipements industriels et matériel d'exploitation, les turbines hydrauliques... Le fonds iconographique illustre la plupart des thèmes abordés par les plans. On y retrouve aussi des collections de photographies prises à l'occasion de visites officielles, mais aussi une série de dessins techniques réalisés par les élèves des écoles schneider. 
Fig.14. Dessin d'élève de l'école Schneider, 1889

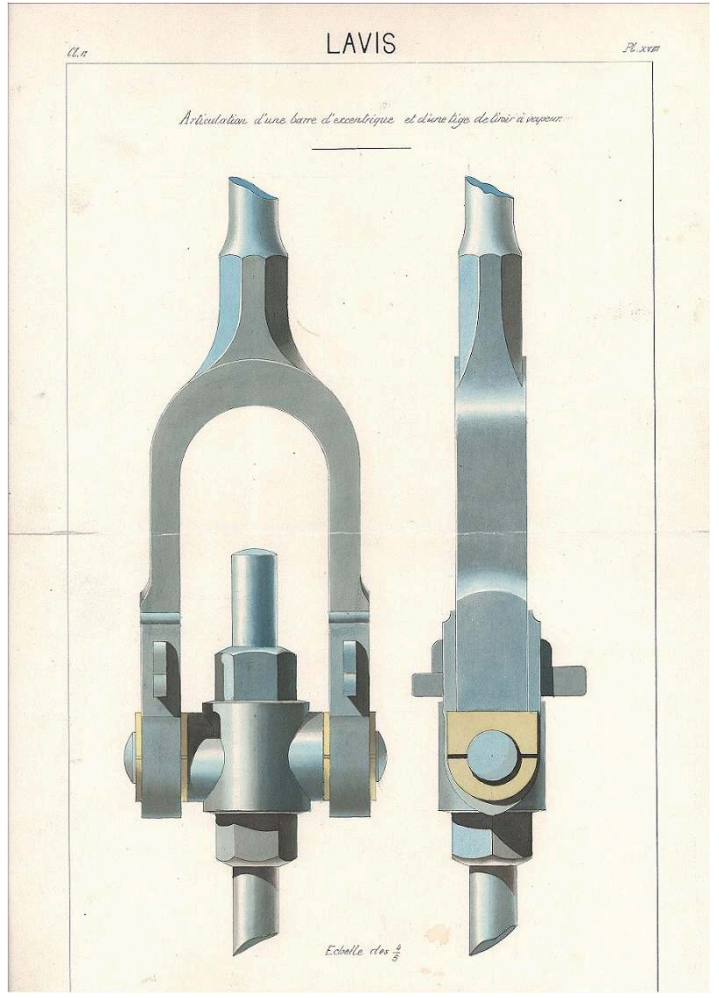

Ce magnifique lavis représente l'articulation d'une barre. Il témoigne du haut niveau de qualification requis.

Centre d'archives de l'Académie François Bourdon, La Verrerie, Le Creusot (Saône-et-Loire)

72 Au-delà des travaux concernant les monographies d'entreprises, de leurs filiales, de leurs unités de production ou de gestion, les fonds couvrent différents secteurs industriels : l'industrie extractive (houillères, carrières, mine de fer, de manganèse...), les industries du feu (sidérurgie, métallurgie, verrerie, céramique...), les constructions mécaniques diverses, les constructions navales, les chemins de fer et le matériel ferroviaire, l'armement (artillerie, chars, munitions, blindages...), la construction métallique (charpentes, ponts, ouvrages d'art...), les bâtiments et travaux publics, la construction électrique et nucléaire, les équipements de production ou de transformation d'énergie (turbines, moteurs, compresseurs...).

\section{BIBLIOGRAPHIE}

Académie François Bourdon, Le métal, la Machine et les Hommes. Deux cents ans d'industrie au Creusot, Le Creusot, 1997

ANGIO Agnès d', Schneider \& Cie et les travaux publics, Paris Genève, Droz, 1995 
ANGIO Agnès d', Schneider et Cie et la naissance de l'ingénierie : des pratiques internes à l'aventure internationale, Paris, CNRS Éditions, 2000

BROISE Tristan de la, TORRÈS Félix, Schneider, l'histoire en force, Paris, Éditions Jean Pierre de Monza, 1996

BEAUD Claude, « Le drame de Creusot-Loire : échec industriel ou fiasco politico-financier ? ", Entreprises et histoire, 2001/1, n²7, p.7-22

BERGERON Louis, Le Creusot, une ville industrielle. Un patrimoine glorieux, Paris, Belin-Herscher, 2001 BRUNO G., Le tour de France par deux enfants, Paris, éd. Belin, 1877, rééd. 1891, 1895, 1906, ..., 1972, 2000

CARTIER Claudine, « Des musées d'art et d'industrie aux musées de site industriel in Autour de l'industrie, histoire et patrimoine ", in BELHOSTE Jean-François, BENOIT Serge, CHASSAGNE Serge, MIOCHE Philipe (dir.), Autour de l'industrie : histoire et patrimoine. Mélanges offerts à Denis Woronoff, Paris, Comité pour l'histoire économique et financière de la France, 2004, p.247-264

CHAUMIER Serge, JACOBI Daniel (dir.), Exposer des idées. Du musée au Centre d'interprétation, Paris, Ed. Complicité, 2009

CORRIAS Pauline, LE FOLL Typhaine, MÖELLO Morgane, « L'Écomusée Creusot Montceau : métaphore d'un modèle exemplaire ", e-Phaïstos, vol. VIII, n 1, 2020 ; DOI : https://doi.org/ 10.4000/ephaistos.7679

DEVILLERS Christian et HUET Bernard, Le Creusot : naissance et développement d'une ville industrielle, 1782-1914, Seyssel, Champ Vallon, 1981

GARIN Aline. « Le Tour de la France par deux enfants de G. Bruno et ses adaptations cinématographiques et télévisuelles », Sociétés \& Représentations, vol.42, n², 2016, p.145-155 ; DOI : https://doi.org/10.3917/sr.042.0145

GARÇON Anne-Françoise, «L'ouvrier ne fait pas patrimoine ", L'archéologie industrielle en France, $n^{\circ} 36,2000$, p.48-59

GRIBET Marie-Françoise, « Les centrales thermiques des mines de La Machine (1893-1923). Une décision de Schneider \& Cie », Bulletin d'histoire de l'électricité, n³3, juin 1999, p.107-123 ; DOI : https://doi.org/10.3406/helec.1999.1437

KHARABA Ivan, « La formation initiale au sein des établissements Schneider au XIX siècle », Revue du Nord, 2003, hors-série $n^{\circ} 17$, p.281-288

KHARABA Ivan, « Les Musées et l'objet industriel : comment une collection d'objets industriels a été constituée ", Revue Musées et collections publiques de France, n²56, 2009

Musée d'Orsay, Les Schneider, Le Creusot. Une famille, une entreprise, une ville (1836-1960), Paris, Librairie Arthème Fayard, Éditions de la Réunion des musées nationaux, 1995

OZOUF Mona, « Le Tour de la France par deux enfants : le petit livre rouge de la République », dans Pierre Nora (dir.), Les Lieux de mémoire, vol. 1, La République, Paris, Gallimard, 1997, p.277-30 PASSAQUI Jean-Philippe, La stratégie des Schneider, du marché à la firme intégrée (1836-1914), Rennes, Presses Universitaires de Rennes, 2006

PASSAQUI Jean-Philippe, Le Creusot, lumières sur la mine, Le Creusot, Académie François Bourdon, 2009 
PASSAQUI Jean-Philippe, «L'approvisionnement en houille des usines du Creusot pendant les guerres de la Révolution et de l'Empire ", Actes du colloque Économie et industrie en Bourgogne, étapes et perspectives, Château-Chinon, 2015, p.195-214

PIQUET Sylvère, TOBELEM Jean-Michel, « Les enjeux du mécénat culturel et humanitaire », Revue Française de gestion, vol.8, n¹67, 2006, p.46-64 ; DOI : https://doi.org/10.3166/rfg.167.49-64

SUZOR Caroline, Le groupe Empain en France. Une saga industrielle et familiale, Bruxelles, Peter Lang, 2016

\section{NOTES}

1. Fondée en 1967, l'International Iron \& Steel Institute poursuit le travail de recherche sur les métaux ferreux qu'avait initié Henry Bessemer dans le cadre de l'Iron \& Steel Institute, à partir de 1869.

2. Pour l'histoire de ce grand groupe industriel, voir Suzor 2016.

3. Pour rappel, le boggie est un « châssis porteur à deux ou trois essieux supportant l'extrémité d'un véhicule de chemin de fer, relié au châssis principal par une articulation » (Dictionnaire Larousse).

4. Désignation des objets pour l'installation d'un musée Schneider dans la Salle du Jeu de Paume. Archives Académie François Bourdon, Cote : salon Schneider 1129-08.

5. Dès leur arrivée au Creusot en 1837, les Schneider mettent en place des écoles d'enseignement général et spécialisé afin d'offrir une formation initiale aux enfants de leurs ouvriers. Jusqu'aux lois Ferry, ces écoles sont publiques et industrielles. Après 1891, les Schneider créent un système scolaire entièrement privé qui se développe en parallèle aux écoles publiques. Sur les écoles, voir Kharaba 2003.

6. Pour l'histoire de cette machine à vapeur et de l'équipement des mines de La Machine, voir Gribet 1999.

7. Le travail sur les messages et la programmation des collections a été réalisé par un petit groupe de scientifiques: Claudine Cartier, Conservateur en chef du patrimoine, spécialiste de l'industrie et des techniques; Denis Woronoff, professeur émérite en histoire économique à l'université Paris 1 Panthéon-Sorbonne, spécialiste en histoire industrielle, Philippe Bourges, ancien directeur du centre de recherche sur les matériaux d'Industeel-France et Ivan Kharaba, commissaire du Pavillon de l'Industrie.

8. Les deux plans en relief ont été réalisés par Digeon fils et Cie. Celui de 1836 fait $215 \mathrm{~cm}$ sur $120 \mathrm{~cm}$, le plan de 1900 fait $650 \mathrm{~cm}$ sur $274 \mathrm{~cm}$. Ils sont composés de bois, de plâtre et de verre.

9. Adolphe et Eugène Schneider achètent l'usine du Creusot en décembre 1836. Ils en deviennent propriétaires le $1^{\text {er }}$ janvier 1837, sous la raison sociale Schneider frères et Compagnie qu'ils créent à cette occasion. En 1845, après le décès d'Adolphe Schneider, la société prend la dénomination Schneider et Compagnie. En 1966, année de sa transformation en société anonyme, la société devient Schneider SA. Aujourd'hui, l'entreprise est un des leaders mondiaux dans la fabrication de matériels électriques. Elle s'intitule Schneider Electric SE.

10. Le tour de France par deux enfants a été utilisé comme livre de lecture dans les écoles jusqu'à l'orée des années 1960. 8,5 millions d'exemplaires ont été vendus entre 1877 et 1977 (Garin 2016 ; Ozouf 1997).

11. Industeel a implanté ses activités de laminage dans l'ancienne grande forge. Framatome occupe les anciens bâtiments des forges et pilons où l'entreprise a une activité de grosse chaudronnerie et d'usinage, notamment pour des pièces destinées au nucléaire. Implanté dans l'ancien atelier d'artillerie nord, Thermodyn GE Baker Hughes fabrique des petites turbines à 
vapeur et des compresseurs centrifuges. Enfin Alstom, installé dans les anciens bâtiments d'artillerie sud, fabrique des boggies, systèmes de roulement des TGV, tramways, métros, etc.

12. Les bustes des maîtres de forge sont ceux d'Adolphe Schneider, sculpté en 1846 par AugustinAlexandre Dumont; d'Eugène Schneider, sculpté par Jules Franceschi en 1870; d'Henri Schneider, sculpté par Émile Peynot en 1924; d'Eugène II Schneider (petit-fils du premier), sculpté par Paul Landowski en 1956 et enfin le buste de Charles Schneider, sculpté aussi par Paul Landowski en 1951.

13. Les statues présentées sont celles sculptées par Henri Bouchard en 1921 : «l'Employé »H. $220 \mathrm{~cm}$; «l'Ouvrier » H. $220 \mathrm{~cm}$ et la statue d'Auguste Cornu, sculptée en 1921, intitulée «Le lamineur au repos » $\mathrm{H} .217 \mathrm{~cm}$.

14. La privatisation du Pavillon de l'Industrie, se fait, sauf exception, en dehors des jours ou des horaires d'ouverture au public.

15. Les entreprises qui ont apporté du mécénat au Pavillon de l'Industrie sont Altead, CréditAgricole-Centre Est, Industeel France, EDF, Framatome (ex Areva-NP), Thermodyn. Le CIEGOS, comité d'établissement inter-entreprises a aussi apporté son soutien financier à la réalisation du centre d'interprétation. Pour des raisons fiscales liées au statut de l'Académie François Bourdon (association reconnue d'intérêt public), il n'est pas possible de facturer la location du Pavillon de l'Industrie. L'Académie François Bourdon réalise déjà un chiffre d'affaires important dans le cadre de ses prestations de gestion d'archives intermédiaires pour le compte d'industriels. La croissance du chiffre d'affaires au regard des subventions et du mécénat placerait l'Académie dans une situation fiscale qui pourrait lui faire perdre son statut d'association reconnue d'intérêt public.

16. Cérémonies de remise des médailles du travail, séminaires, réunion de comités centraux d'établissement, moment conviviaux...

17. http://www.pavillon-industrie.fr/connexion-enseignant.

18. http://statistiques.pole-emploi.org/bmo/.

19. https://www.pavillon-industrie.fr/pole-archives-documentation .

20. Toutes les archives conservées par l'Académie François Bourdon sont consultables dans la limite des délais de communicabilité. Le centre d'archives accueille les chercheurs du lundi au vendredi, de 8 h30 à 17 h00.

\section{RÉSUMÉS}

L'Académie François Bourdon, association loi 1901, a été créée en 1985 par des anciens cadres de l'usine du Creusot du groupe Creusot-Loire. Cette année-là, au moment de la liquidation de Creusot-Loire, d'anciens salariés se préoccupent de la sauvegarde des archives de la société qui referment non seulement les archives produites entre 1970 et 1985, mais aussi les fonds plus anciens remontant à la création de la société Schneider en 1837. Quelques documents portent même sur la période précédente depuis le début de la Fonderie Royale et de l'histoire de la métallurgie au Creusot, en 1782. Forte de son fonds initial, l'Académie François Bourdon est vite reconnue comme centre d'archives industrielles. Elle gère aussi une collection d'objets patrimoniaux d'environ 1200 pièces dont une partie est présentée au centre d'interprétation du Pavillon de l'Industrie sur le site du Château de la Verrerie au Creusot. 
The Académie François Bourdon, an association under the law of 1901, was created in 1985 by former managers of the Creusot-Loire group's factory in Le Creusot. That year, when CreusotLoire went into liquidation, the former employees became concerned about safeguarding the company's archives, which included not only the archives produced between 1970 and 1985, but also older collections dating back to the creation of the Schneider company in 1837. That year, when Creusot-Loire went into liquidation, the former employees became concerned about safeguarding the company's archives, which included not only the archives produced between 1970 and 1985, but also older collections dating back to the creation of the Schneider company in 1837. Some documents even concern the earlier period: the beginnings of the Royal Foundry and the history of metallurgy in Le Creusot from 1782. With this initial collection, the Académie François Bourdon was quickly recognized as a center for industrial archives. It also manages a collection of heritage objects of about 1200 items, some of which are exhibited in the interpretation center of the "Pavillon de l'Industrie" which is located on the site of the Château de la Verrerie in Le Creusot.

\section{INDEX}

Mots-clés : histoire des techniques, archive, musée technique, industrie, patrimoine industriel

Keywords : history of technology, archive, technical museum, industry, industrial heritage

\section{AUTEUR}

\section{IVAN KHARABA}

Directeur de l'Académie François Bourdon au Creusot, Ivan Kharaba a soutenu sa thèse sur l'« Histoire de la Chambre de commerce et d'industrie du Var : l'action consulaire (1833-1896) », à l'Université Aix-Marseille 1. Ses principales publications portent sur l'histoire industrielle, les patrimoines issus de l'industrie et leur valorisation. 